\title{
ON CERTAIN HEUN FUNCTIONS, ASSOCIATED FUNCTIONS OF DISCRETE VARIABLES, AND APPLICATIONS
}

\author{
P. MALITS
}

Received 15 November 2002

\begin{abstract}
Two sets of the Heun functions are introduced via integrals. Theorems about expanding functions with respect to these sets are proven. A number of integral and series representations as well as integral equations and asymptotic formulas are obtained for these functions. Some of the coefficients of the series are orthogonal (J-orthogonal) functions of discrete variables and may be interpreted as orthogonal polynomials. Other sets of the coefficients are biorthonormal. Expanding infinite vectors to series with respect to the coefficients is discussed. Certain Legendre functions of complex degree are limiting cases of the studied functions. This leads to new relations for Legendre functions and associated integral transforms. The treated Heun functions find a use for solving dual Fourier series equations which are reduced to the Fredholm integral equations of the second kind. Explicit solutions are obtained in a special case.
\end{abstract}

2000 Mathematics Subject Classification: 33E20, 33C47, 42C10, 45F10.

1. Introduction. We consider in this paper the functions $P n_{m}\left(x \mid k^{2}\right)=$ $P n_{m}(x)$ and $\widetilde{P} n_{m}\left(x \mid k^{2}\right)=\widetilde{P} n_{m}(x)$ which are defined for $k^{2}<1$ via the integral representations

$$
\begin{aligned}
P n_{m}\left(x \mid k^{2}\right) & =\int_{0}^{x} \frac{\mathrm{cn}(t / 2) \cos \left(\mu_{m} t\right)}{\sqrt{\mathrm{cn}^{2}(t / 2)-\mathrm{cn}^{2}(x / 2)}} d t, \\
\widetilde{P} n_{m}\left(x \mid k^{2}\right) & =\frac{(-1)^{m}}{\operatorname{dn}(x / 2)} P n_{m}\left(2 \mathbf{K}-x \mid k^{2}\right) \\
& =\int_{x}^{2 \mathbf{K}} \frac{\operatorname{sn}(t / 2) \cos \left(\mu_{m} t\right)}{\sqrt{\mathrm{cn}^{2}(x / 2)-\mathrm{cn}^{2}(t / 2)}} d t,
\end{aligned}
$$

where $0 \leq x \leq 2 \mathbf{K}, \mathbf{K}=\mathbf{K}(k)$ is the complete elliptic integral of the first kind, $\operatorname{sn} x=\operatorname{sn}(x \mid k), \operatorname{cn} x=\operatorname{cn}(x \mid k)$, and $\operatorname{dn} x=\operatorname{dn}(x \mid k)$ are the Jacobian elliptic functions, and $\mu_{m}=\pi m / 2 \mathbf{K}$.

Originally our investigation was motivated by applications of the treated functions for solving some dual series equations arising in various periodic mixed boundary value problems (see Section 9). But it appeared that the subject arouses independent interest. It is shown in Section 7 that the above functions are certain Heun functions. They are the only instances of the Heun 
functions known to the author which are given explicitly and have simple integral representations (Section 1). The integral representations enable us to obtain series expansions for $P n_{m}(x)$ (Sections 2 and 5) as well as asymptotic formulas (Section 4) and integral equations (Section 6). The coefficients of these series constitute orthogonal (J-orthogonal or biorthogonal) bases in certain sequence spaces (Sections 3 and 5). Some of them are orthogonal polynomials. This is useful for analytical and numerical calculations of $P n_{m}(x)$-series as well as Fourier series. The theorems about the expansion of an arbitrary function into the $P n_{m}(x)$-series established in Section 5 give the first examples of such conditions of validity for expanding in the Heun functions that are not covered by the general Sturm-Liouville theory [9, page 62]. The completeness in $L_{2}$ is proven. The convergence of the Abel means is shown for functions belonging to $L_{1}$. The absolute convergence of the expansions for functions from $L_{1}$ exists under additional restrictions. The problem of efficient calculation of the coefficients is also discussed.

Employing the well-known properties of the Jacobian elliptic functions and the elliptic integrals $[5,11]$, one can show that

$$
\begin{aligned}
& P n_{m}\left(x k^{\prime} \mid-\frac{k^{2}}{k^{\prime 2}}\right)=P n_{m}\left(x \mid k^{2}\right) \operatorname{dn}(x \mid k), \quad k^{\prime}=\sqrt{1-k^{2}} \\
& \widetilde{P} n_{m}\left(x k^{\prime} \mid-\frac{k^{2}}{k^{\prime 2}}\right)=k^{\prime} \widetilde{P} n_{m}\left(x \mid k^{2}\right) \operatorname{dn}(x \mid k) .
\end{aligned}
$$

For this reason, we will further assume that $0<k<1$. In the limiting cases, the functions introduced above may be expressed in terms of Jacobi polynomials as $k=0$ and Legendre functions of complex degree as $k=1, \mu_{m}=\xi$. In the last case, a number of new relations are found for the Mehler-Fok transform and another related integral transform (Section 8). The corresponding series coefficients become the Pollaczek polynomials.

Our first objective is to deduce new integral representations. To achieve that, we examine the contour integral

$$
\oint_{L}\left(1-\frac{\mathrm{cn}^{2}(x / 2)}{\operatorname{cn}^{2}(z / 2)}\right)^{-1 / 2} \exp \left( \pm i \mu_{m} z\right) d z=0, \quad x>0 .
$$

The closed contour $L$ consists of arcs of small radii $|z \pm x|=\varepsilon$ and $|z \pm 2 \mathbf{K}|=\varepsilon$ lying within the upper half-plane and connecting segments of the rectangle having vertices $z= \pm 2 \mathbf{K}$ and $z= \pm 2 \mathbf{K}+2 i \mathbf{K}\left(k^{\prime}\right)$. The integrand is an analytic function in the domain bounded by $L$ and hence the integral is zero. Because the line integrals along the vertical segments cancel each other, we find that, as $\varepsilon \rightarrow 0$, 


$$
\begin{aligned}
P n_{m}(x)= & \exp (\mp \lambda m) \int_{0}^{2 \mathrm{~K}} \frac{\mathrm{dn}(t / 2)}{\sqrt{k^{2} \mathrm{cn}^{2}(x / 2) \operatorname{sn}^{2}(t / 2)+\mathrm{dn}^{2}(t / 2)}} \cos \left(\mu_{m} t\right) d t \\
& \pm \int_{x}^{2 \mathbf{K}} \frac{\mathrm{cn}(t / 2)}{\sqrt{\mathrm{cn}^{2}(x / 2)-\mathrm{cn}^{2}(t / 2)}} \sin \left(\mu_{m} t\right) d t
\end{aligned}
$$

where $\lambda=\pi \mathbf{K}\left(k^{\prime}\right) / \mathbf{K}(k)$. Finally, we obtain the representations

$$
\begin{aligned}
\operatorname{Pn}_{m}(x) & =\cosh (\lambda m) \int_{0}^{2 \mathrm{~K}} \frac{\mathrm{dn}(t / 2) \cos \left(\mu_{m} t\right)}{\sqrt{k^{2} \mathrm{cn}^{2}(x / 2) \mathrm{sn}^{2}(t / 2)+\mathrm{dn}^{2}(t / 2)}} d t \\
& =\cosh (\lambda m) \int_{0}^{2 \mathrm{~K}} \frac{\mathrm{dn}(t / 2) \cos \left(\mu_{m} t\right)}{\sqrt{1-k^{2} \mathrm{sn}^{2}(x / 2) \mathrm{sn}^{2}(t / 2)}} d t \\
\operatorname{Pn}_{m}(x) & =\operatorname{coth}(\lambda m) \int_{x}^{2 \mathrm{~K}} \frac{\mathrm{cn}(t / 2) \sin \left(\mu_{m} t\right)}{\sqrt{\mathrm{cn}^{2}(x / 2)-\mathrm{cn}^{2}(t / 2)}} d t .
\end{aligned}
$$

Corresponding formulas for $\widetilde{P} n_{m}$ ( $x$ ) follow from (1.6), (1.7), and (1.2) by changing from $x$ to $2 \mathbf{K}-x$ and from $t$ to $2 \mathbf{K}-t$ :

$$
\begin{aligned}
& \tilde{P} n_{m}(x)=\cosh (\lambda m) \int_{0}^{2 \mathrm{~K}} \frac{\cos \left(\mu_{m} t\right)}{\sqrt{1-k^{2} \mathrm{sn}^{2}(x / 2) \mathrm{sn}^{2}(t / 2)}} d t, \\
& \tilde{P} n_{m}(x)=-\operatorname{coth}(\lambda m) \int_{0}^{x} \frac{\operatorname{sn}(t / 2) \sin \left(\mu_{m} t\right)}{\sqrt{\mathrm{cn}^{2}(t / 2)-\mathrm{cn}^{2}(x / 2)}} d t .
\end{aligned}
$$

In the foregoing, the standard relations of Jacobian elliptic functions have been used.

Now let $x$ be a complex number such that $k^{2} \operatorname{sn}^{2}(x / 2)$ is not a real number between 1 and $\infty$. Then the integrals in (1.6) and (1.8) remain convergent and define analytic functions on the appropriate Riemann surface. Consequently, these integrals may be employed to analytically extend $P n_{m}(x)$ and $\widetilde{P} n_{m}(x)$ to the cut complex plane.

Several other representations can be found by integration by parts of certain integrals. Two of them are given by

$$
\begin{aligned}
& \frac{\pi m \operatorname{sn}(x / 2)}{2 \mathbf{K c d}(x / 2)} P n_{m}(x)=\frac{d}{d x} \int_{0}^{x} \frac{\operatorname{sn}(t / 2) \mathrm{dn}(t / 2) \sin \left(\mu_{m} t\right)}{\sqrt{\mathrm{cn}^{2}(t / 2)-\mathrm{cn}^{2}(x / 2)}} d t, \quad \operatorname{cd} x=\frac{\operatorname{cn} x}{\operatorname{dn} x}, \\
& \frac{\pi m \operatorname{sn}(x / 2)}{2 \mathbf{K c d}(x / 2)} \tanh (\lambda m) P n_{m}(x)=-\frac{d}{d x} \int_{x}^{2 \mathbf{K}} \frac{\operatorname{sn}(t / 2) \operatorname{dn}(t / 2) \cos \left(\mu_{m} t\right)}{\sqrt{\mathrm{cn}^{2}(x / 2)-\mathrm{cn}^{2}(t / 2)}} d t .
\end{aligned}
$$

There are analogous formulas for $\widetilde{P} n_{m}(x)$ as well. 
2. Expansions in powers of the Jacobian elliptic functions. The simplest series expansion of $P n_{m}(x)$ in ascending powers of Jacobian elliptic functions is derived from (1.6) by substituting the binomial series for the inverse square root. Subsequent term-by-term integration casts $P n_{m}(x)$ into the form

$$
P n_{m}(x)=\pi \sum_{n=0}^{\infty} \alpha_{m n} \frac{(2 n-1) ! !}{(2 n) ! !}\left(\operatorname{sn} \frac{x}{2}\right)^{2 n}
$$

where $k|\operatorname{sn}(x / 2)|<1,0 ! !=(-1) ! !=1$, and the coefficients $\alpha_{m n}$ are given by

$$
\alpha_{m n}=\frac{k^{2 n} \cosh (\lambda m)}{\pi} \int_{0}^{2 \mathrm{~K}} \operatorname{dn} \frac{t}{2}\left(\operatorname{sn} \frac{t}{2}\right)^{2 n} \cos \left(\mu_{m} t\right) d t
$$

We will also use below the coefficients $\alpha_{0 n}$ which are evaluated in a closed form:

$$
\alpha_{0 n}=\frac{k^{2 n}(2 n-1) ! !}{(2 n) ! !}
$$

The corresponding series (2.1) in this case defines the function $P n_{0}(x)=$ $2 \mathbf{K}(k \operatorname{sn}(x / 2))$. Note that the coefficients (2.2) may be considered as moments of the trigonometric function.

We seek an expansion of $\cos \left(\mu_{m} t\right)$ in powers of the Jacobian elliptic functions. We may interpret (1.1) as the Abel integral equation. Its solution is

$$
\cos \left(\mu_{m} t\right)=\frac{1}{\pi \mathrm{cn}(t / 2)} \frac{d}{d t} \int_{0}^{t} \frac{P n_{m}(x) d\left(\mathrm{sn}^{2}(x / 2)\right)}{\sqrt{\operatorname{sn}^{2}(t / 2)-\mathrm{sn}^{2}(x / 2)}} .
$$

Making the substitution (2.1) and integrating term by term, this relation becomes

$$
\cos \left(\mu_{m} t\right)=\operatorname{dn} \frac{t}{2} \sum_{n=0}^{\infty} \alpha_{m n}\left(\operatorname{sn} \frac{t}{2}\right)^{2 n}
$$

By evaluating the binomial series, we ascertain that (2.5) remains valid for $m=0$ as well.

The following three-term recurrence formula is obtained as a result of acting by the trigonometric differential operator $d^{2} / d t^{2}+\mu_{m}^{2}$ on (2.5) and equating coefficients of like powers:

$$
\begin{aligned}
& 2 n(2 n-1) \alpha_{m n}+\left[4 \mu_{m}^{2}-k^{2}(2 n-1)^{2}-4(n-1)^{2}\right] \alpha_{m, n-1} \\
& \quad+2(n-1)(2 n-3) k^{2} \alpha_{m, n-2}=0 .
\end{aligned}
$$

Two initial coefficients $\alpha_{m 0}=1$ and $\alpha_{m 1}=(1 / 2)\left(k^{2}-4 \mu_{m}^{2}\right)$ are directly evaluated from (2.2). One may take $\alpha_{m,-1}=0$ and $\alpha_{m 0}=1$ instead of them. 
In an analogous manner, we obtain from the integral representation (1.8) that

$$
\widetilde{P} n_{m}(x)=-\frac{\pi^{2} m}{\mathbf{K}} \operatorname{coth} \lambda m \sum_{n=1}^{\infty} \beta_{m n} \frac{(2 n-1) ! !}{(2 n) ! !}\left(\operatorname{sn} \frac{x}{2}\right)^{2 n}
$$

where $k|\operatorname{sn}(x / 2)|<1$ and

$$
\begin{aligned}
\beta_{m n} & =-\frac{k^{2 n} \sinh (\lambda m)}{2 \pi \mu_{m}} \int_{0}^{2 \mathbf{K}}\left(\operatorname{sn} \frac{t}{2}\right)^{2 n} \cos \left(\mu_{m} t\right) d t \\
& =\frac{k^{2 n} n \sinh (\lambda m)}{2 \pi \mu_{m}^{2}} \int_{0}^{2 \mathbf{K}} \operatorname{cn} \frac{t}{2} \operatorname{dn} \frac{t}{2}\left(\operatorname{sn} \frac{t}{2}\right)^{2 n-1} \sin \left(\mu_{m} t\right) d t .
\end{aligned}
$$

Therefore, the corresponding expansion of $P n_{m}(x)$ is given by

$$
P n_{m}(x)=\frac{(-1)^{m+1} \pi^{2} m k^{\prime} \operatorname{coth}(\lambda m)}{\mathrm{Kdn}(x / 2)} \sum_{n=1}^{\infty} \beta_{m n} \frac{(2 n-1) ! !}{(2 n) ! !}\left(\operatorname{cd} \frac{x}{2}\right)^{2 n} \text {. }
$$

The numbers $\beta_{m n}$ obey the three-term recurrence formula

$$
\begin{aligned}
& (n-1)(2 n-1) \beta_{m n}+2\left[\mu_{m}^{2}-(n-1)^{2}\left(1+k^{2}\right)\right] \beta_{m, n-1} \\
& +(n-1)(2 n-3) k^{2} \beta_{m, n-2}=0, \quad \beta_{m 0}=0, \beta_{m 1}=1,
\end{aligned}
$$

where $\beta_{m 1}$ is evaluated from Fourier series for $(\operatorname{sn}(t / 2))^{2}$ [11]. These numbers are the coefficients of the series representation of $\sin \left(\mu_{m} t\right)$ in powers of Jacobian elliptic functions:

$$
\sin \left(\mu_{m} t\right)=2 \mu_{m} \operatorname{cn} \frac{t}{2} \operatorname{dn} \frac{t}{2} \sum_{n=1}^{\infty} \beta_{m n}\left(\operatorname{sn} \frac{t}{2}\right)^{2 n-1}
$$

or, equivalently,

$$
\cos \left(\mu_{m} t\right)=1-2 \mu_{m}^{2} \sum_{n=1}^{\infty} \frac{1}{n} \beta_{m n}\left(\operatorname{sn} \frac{t}{2}\right)^{2 n}
$$

Upon equating (2.5) and (2.12), we establish formulas expressing one type of the coefficients in terms of the other:

$$
\begin{aligned}
& \alpha_{m n}=\alpha_{0 n}-2 \mu_{m}^{2}\left[\frac{1}{n} \beta_{m n}+\sum_{l=1}^{n-1} \frac{k^{2 l}(2 l-1) ! !}{(n-l)(2 l) ! !} \beta_{m, n-l}\right] \\
& \beta_{m n}=\frac{n}{2 \mu_{m}^{2}}\left[\frac{k^{2}}{2} \alpha_{m, n-1}-\alpha_{m n}-\sum_{l=2}^{n} \frac{k^{2 l}(2 l-3) ! !}{(2 l) ! !} \alpha_{m, n-l}\right] .
\end{aligned}
$$


Two additional series for $P n_{m}(x)$ result from (1.6) and (1.8):

$$
\begin{aligned}
& P n_{m}(x)=\frac{\pi}{\operatorname{dn}(x / 2)} \sum_{n=0}^{\infty} \gamma_{m n} \frac{(2 n-1) ! !}{(2 n) ! !}\left(k^{\prime} \operatorname{sd} \frac{x}{2}\right)^{2 n}, \quad \operatorname{sn} \frac{x}{2}<\frac{1}{\sqrt{2} k} \\
& P n_{m}(x)=\frac{(-1)^{m+1} \pi^{2} m}{\left(k^{\prime}\right)^{2} \mathbf{K} \tanh (\lambda m)} \sum_{n=1}^{\infty} \zeta_{m n} \frac{(2 n-1) ! !}{(2 n) ! !}\left(\operatorname{cn} \frac{x}{2}\right)^{2 n}, \quad \text { cn } \frac{x}{2}<\frac{k^{\prime}}{k}
\end{aligned}
$$

Here we use the notations

$$
\begin{aligned}
\gamma_{m n} & =\frac{(-1)^{n}}{\pi}\left(\frac{k}{k^{\prime}}\right)^{2 n} \cosh (\lambda m) \int_{0}^{2 \mathbf{K}} \operatorname{dn} \frac{t}{2}\left(\operatorname{cn} \frac{t}{2}\right)^{2 n} \cos \left(\mu_{m} t\right) d t \\
\frac{(-1)^{n}}{\sinh (\lambda m)} \zeta_{m n} & =\frac{k^{\prime}}{2 \pi \mu_{m}}\left(\frac{k}{k^{\prime}}\right)^{2 n} \int_{0}^{2 \mathbf{K}}\left(\operatorname{cn} \frac{t}{2}\right)^{2 n} \cos \left(\mu_{m} t\right) d t \\
& =\frac{k^{\prime} n}{2 \pi \mu_{m}^{2}}\left(\frac{k}{k^{\prime}}\right)^{2 n} \int_{0}^{2 \mathbf{K}} \operatorname{sn} \frac{t}{2} \operatorname{dn} \frac{t}{2}\left(\operatorname{cn} \frac{t}{2}\right)^{2 n-1} \sin \left(\mu_{m} t\right) d t
\end{aligned}
$$

The coefficients $\gamma_{m n}$ and $\zeta_{m n}$ obey the recurrence relations (2.6) and (2.10), respectively, where $k$ is changed to $i k / k^{\prime}\left(\mathbf{K}\right.$ to $\left.\mathbf{K}\left(i k / k^{\prime}\right)=k^{\prime} \mathbf{K}\right)$. These coefficients give rise to two new series expansions of trigonometric functions in powers of Jacobian elliptic functions:

$$
\begin{aligned}
& \cos \left(\mu_{m} t\right)=\frac{(-1)^{m} \operatorname{dn}(t / 2)}{k^{\prime}} \sum_{n=0}^{\infty} \gamma_{m n}\left(\operatorname{cn} \frac{t}{2}\right)^{2 n}, \quad \operatorname{sn} \frac{t}{2}<\frac{1}{\sqrt{2} k}, \\
& \sin \left(\mu_{m} t\right)=\frac{2(-1)^{m+1} \mu_{m}}{\left(k^{\prime}\right)^{2}} \operatorname{sn} \frac{t}{2} \operatorname{dn} \frac{t}{2} \sum_{n=1}^{\infty} \zeta_{m n}\left(\operatorname{cn} \frac{t}{2}\right)^{2 n}, \quad \operatorname{cn} \frac{t}{2}<\frac{k^{\prime}}{k} .
\end{aligned}
$$

Direct links between $\gamma_{m n}$ and $\zeta_{m n}$ are given by the relations (2.13), where $\alpha_{m n}=\gamma_{m n}, \beta_{m n}=\zeta_{m n}$, and $k$ is changed into $i k / k^{\prime}$ (K into $\left.k^{\prime} \mathbf{K}\right)$.

Nonlinear relations between the coefficients over indices $m$ may be found by replacing the trigonometric functions in formulas for $\sin \left[\left(\mu_{m}+\mu_{r}\right) t\right]$ and $\cos \left[\left(\mu_{m}+\mu_{r}\right) t\right]$ with their expansions and comparing coefficients of like powers. For example, as $m=r$, we read

$$
\beta_{2 m, n}=\beta_{m, n}-2 \mu_{m}^{2} \sum_{l=1}^{n-1} \frac{1}{l} \beta_{m, l} \beta_{m, n-l} .
$$

3. Orthogonality of coefficients. The orthogonality property of the coefficients $\alpha_{m n}$ may be deduced from the generating function (2.5) by using their 
definition (2.2) and the orthogonality property of $\cos \left(\mu_{m} t\right)$ :

$$
\frac{\pi}{\mathbf{K} \cosh (\lambda m)} \sum_{n=0}^{\infty} \frac{\alpha_{m n} \alpha_{l n}}{k^{2 n}}=\varepsilon_{l} \delta_{l m}, \quad m, l=0,1, \ldots,
$$

where $\varepsilon_{l}=1$ for $l \neq 0$, and $\varepsilon_{l}=2$ for $l=0$ and $\delta_{l m}$ is the Kronecker delta.

One can see from (2.2) that $\alpha_{m n}$ are Fourier coefficients of a certain function. This implies that

$$
\frac{1}{2} \alpha_{0 r}+\sum_{m=1}^{\infty} \frac{\alpha_{m r}}{\cosh (\lambda m)} \cos \frac{\pi m t}{2 \mathbf{K}}=\frac{\mathbf{K} k^{2 r}}{\pi} \operatorname{dn} \frac{t}{2}\left(\operatorname{sn} \frac{t}{2}\right)^{2 r}
$$

Inserting the series (2.5) gives

$$
\sum_{n=0}^{\infty} \operatorname{dn} \frac{t}{2}\left(\operatorname{sn} \frac{t}{2}\right)^{2 n}\left[\frac{1}{2} \alpha_{0 n} \alpha_{0 r}+\sum_{m=1}^{\infty} \frac{\alpha_{m n} \alpha_{m r}}{\cosh (\lambda m)}\right]=\frac{\mathbf{K} k^{2 r}}{\pi} \operatorname{dn} \frac{t}{2}\left(\operatorname{sn} \frac{t}{2}\right)^{2 r} .
$$

Hence we obtain another orthogonality property

$$
\frac{\pi}{\mathbf{K} k^{2 r}} \sum_{m=0}^{\infty} \frac{\alpha_{m n} \alpha_{m r}}{\varepsilon_{m} \cosh (\lambda m)}=\delta_{n r}, \quad n, r=0,1, \ldots
$$

In the same way

$$
\begin{gathered}
\frac{\pi^{4} m^{3}}{2 \mathbf{K}^{4} \sinh (\lambda m)} \sum_{n=1}^{\infty} \frac{\beta_{m n} \beta_{l n}}{n k^{2 n}}=\delta_{l m}, \quad m, l=1,2, \ldots, \\
\frac{\pi^{4}}{2 k^{2 n} \mathbf{K}^{4} n} \sum_{m=1}^{\infty} \frac{m^{3} \beta_{m n} \beta_{m r}}{\sinh (\lambda m)}=\delta_{n r}, \quad n, r=1,2, \ldots
\end{gathered}
$$

These relations show that the infinite matrices $\left\{\alpha_{m n}\right\}$ and $\left\{\beta_{m n}\right\}$ are orthogonal matrices. As $k<\sqrt{2} / 2$, analogous formulas for $\gamma_{m n}$ and $\zeta_{m n}$ are of the form

$$
\begin{gathered}
\frac{\pi}{k^{\prime} \mathbf{K} \cosh (\lambda m)} \sum_{n=0}^{\infty}(-1)^{m+n}\left(\frac{k^{\prime}}{k}\right)^{2 n} \gamma_{m n} \gamma_{l n}=\varepsilon_{l} \delta_{l m}, \quad m, l=0,1, \ldots, \\
\frac{\pi}{k^{\prime} \mathbf{K}}\left(\frac{k^{\prime}}{k}\right)^{2 n} \sum_{m=0}^{\infty}(-1)^{m+n} \frac{\gamma_{m n} \gamma_{m r}}{\varepsilon_{m} \cosh (\lambda m)}=\delta_{n r}, \quad n, r=0,1, \ldots, \\
\frac{\pi^{4} m^{3}}{2\left(k^{\prime} \mathbf{K}\right)^{4} \sinh (\lambda m)} \sum_{n=1}^{\infty} \frac{(-1)^{m+n}}{n}\left(\frac{k^{\prime}}{k}\right)^{2 n} \zeta_{m n} \zeta_{l n}=\delta_{l m}, \quad m, l=1,2, \ldots, \\
\frac{\pi^{4}}{2 k^{2 n}\left(k^{\prime} \mathbf{K}\right)^{4} n}\left(\frac{k^{\prime}}{k}\right)^{2 n} \sum_{m=1}^{\infty}(-1)^{m+n} \frac{m^{3} \zeta_{m n} \zeta_{m r}}{\sinh (\lambda m)}=\delta_{n r}, \quad n, r=1,2, \ldots
\end{gathered}
$$

It is seen that $\gamma_{m n}$ and $\zeta_{m n}$ are elements of the J-orthogonal matrices.

Thus the coefficients of the expansions indicated above can be interpreted as some functions of two discrete variables associated with $P n_{m}(x)$. They are 
orthogonal (J-orthogonal) over each of the variables. Especially, we observe from the recurrence relations that these functions are orthogonal polynomials in the variable $\mu_{m}^{2}$. There is no source of reference for these polynomials known to the author.

The orthogonality relations generate formulas for developments of an arbitrary infinite vector $\mathbf{F}\left(a_{0}, a_{1}, \ldots\right)$ into linear combinations of vectors of the orthogonal (J-orthogonal) matrices mentioned above. For example, the expansions

$$
\begin{aligned}
& a_{m}=\sum_{n=0}^{\infty} \tilde{a}_{n} \alpha_{m n}, \quad m=0,1, \ldots, \\
& \tilde{a}_{n}=\frac{\pi}{\mathbf{K} k^{2 n}} \sum_{m=0}^{\infty} \frac{a_{m} \alpha_{m n}}{\varepsilon_{m} \cosh (\lambda m)}, \quad n=0,1, \ldots,
\end{aligned}
$$

are trivial as one of the sequences $a_{m}$ or $\tilde{a}_{n}$ is finite. In general, such developments are valid for vectors belonging to certain special spaces. We commence with a negative result demonstrating that rather restrictive conditions should be imposed on $\tilde{a}_{n}$.

THEOREM 3.1. The set of the vectors $\left\{k^{-n} \alpha_{m n}\right\}_{n=0}^{\infty}, m=0,1, \ldots$, is not a basis in the space $l_{2}$.

PROof. A starting point is the equation

$$
\begin{gathered}
\xi_{m} k^{-n} \alpha_{m n}=\sum_{r=0}^{\infty} A_{n+r} k^{-r} \alpha_{m r}, \quad n=0,1, \ldots, \xi_{m}=\frac{\pi}{\varepsilon_{m} \cosh (\lambda m)}, \\
A_{n}=k^{n} \int_{0}^{2 \mathrm{~K}} \operatorname{dn}^{2} \frac{t}{2}\left(\operatorname{sn} \frac{t}{2}\right)^{2 n} d t=\frac{k^{2 n}(2 n-1) ! !}{\pi(2 n) ! !} F\left(-\frac{1}{2}, n+\frac{1}{2}, n+1, k^{2}\right),
\end{gathered}
$$

where $F(\alpha, \beta, \gamma, x)$ is the hypergeometric function. This equation is derived from formulas (2.5) and (2.2). It is readily seen that the vector $\left\{k^{-n} \alpha_{m n}\right\}_{n=0}^{\infty}$ is the eigenvector which corresponds to the eigenvalue $\lambda_{m}=\xi_{m}$ of the positive selfadjoint matrix operator A. The operator A has the finite trace

$$
\begin{aligned}
\operatorname{tr} \mathbf{A} & =\sum_{n=0}^{\infty} k^{2 n} \int_{0}^{2 \mathbf{K}} \operatorname{dn}^{2} \frac{t}{2}\left(\operatorname{sn} \frac{t}{2}\right)^{4 n} d t=\int_{0}^{2 \mathbf{K}} \frac{\mathrm{dn}^{2}(t / 2)}{1-k^{2} \operatorname{sn}^{4}(t / 2)} d t \\
& =\mathbf{K}+\int_{0}^{2 \mathbf{K}} \frac{\left(1-k \operatorname{sn}^{2}(t / 2)\right)^{2}+2 k(1-k) \operatorname{sn}^{2}(t / 2)}{2\left(1-k^{2} \operatorname{sn}^{4}(t / 2)\right)} d t
\end{aligned}
$$

which is equal to the sum of all eigenvalues. On the other hand,

$$
\sum_{m=0}^{\infty} \xi_{m}=\frac{\pi}{2}+\sum_{m=1}^{\infty} \frac{\pi}{\cosh (\lambda m)}=\mathbf{K} \operatorname{dn}(0)=\mathbf{K}<\operatorname{tr} \mathbf{A} .
$$

Then $\xi_{m}, m=0,1, \ldots$, do not form the complete set of the eigenvalues of the operator A which is completely continuous in $l_{2}$. Hence, the set of the corresponding eigenvectors is incomplete as well. 
The trivial sufficient condition arises from evident estimates

$$
\left|\alpha_{m n}\right| \leq \frac{2 \mathbf{K}}{\pi} k^{2 n} \cosh (\lambda m), \quad\left|\frac{\alpha_{m n} \alpha_{m r}}{\cosh (\lambda m)} \tilde{a}_{r}\right| \leq \frac{\alpha_{m n}^{2}+\alpha_{m r}^{2}}{2 \cosh (\lambda m)}\left|\tilde{a}_{r}\right| .
$$

THeOREM 3.2. Suppose $\tilde{a}_{n} \in l_{1}$. If $a_{m}$ is defined by (3.7), then the inversion formula (3.8) is valid. Series in these formulas converge absolutely.

The important example is the inversion of the series (2.1), namely,

$$
\frac{(2 n-1) ! !}{(2 n) ! !}\left(k \operatorname{sn} \frac{x}{2}\right)^{2 n}=\frac{1}{\mathbf{K}} \sum_{m=0}^{\infty} \frac{\alpha_{m n}}{\varepsilon_{m} \cosh (\lambda m)} P n_{m}(x), \quad x \neq 2 \mathbf{K} .
$$

This formula does not hold at the point $x=2 \mathbf{K}$ since in this case $\tilde{a}_{n}=(2 n-$ $1) ! ! /(2 n) ! ! \notin l_{1}$.

THEOREM 3.3. Let $a_{m}$ be Fourier coefficients of an even function $g(t) \in$ $L_{1}(-2 \mathbf{K}, 2 \mathbf{K})$. If $\tilde{a}_{n}$ is defined by (3.8), then the inversion formula (3.7) is valid and, moreover,

$$
\tilde{a}_{n}=\frac{1}{\mathbf{K}} \int_{0}^{2 \mathbf{K}} \operatorname{dn} \frac{t}{2}\left(\operatorname{sn} \frac{t}{2}\right)^{2 n} g(t) d t
$$

Proof. The relation (3.14) is obtained on the basis of (3.2) by applying the Parseval formula for the set $\left\{\cos \left(\mu_{m} t\right)\right\}$ to the convergent series (3.8). Substituting (3.14) and interchanging the order of summation and integration, we find

$$
\begin{aligned}
\sum_{n=0}^{\infty} \tilde{a}_{n} \alpha_{m n} & =\frac{1}{\mathbf{K}} \int_{0}^{2 \mathbf{K}}\left[\operatorname{dn} \frac{t}{2} \sum_{n=0}^{\infty} \alpha_{m n}\left(\operatorname{sn} \frac{t}{2}\right)^{2 n}\right] g(t) d t \\
& =\frac{1}{\mathbf{K}} \int_{0}^{2 \mathbf{K}} g(t) \cos \left(\mu_{m} t\right) d t=a_{m} .
\end{aligned}
$$

This completes our proof.

We derive the following corollary from this theorem by referring to RieszFisher's theorem.

COROLLARY 3.4. Formulas (3.7) and (3.8) are valid as $a_{m} \in l_{2}$.

A proof for a more general sequence space may be done by very elementary arguments.

THEOREM 3.5. Let the series

$$
\sum_{m=0}^{\infty} \frac{\left|a_{m}\right|^{2}}{\cosh (\lambda m)}
$$

be convergent and let $\tilde{a}_{n}$ be defined by (3.8). Then the formula (3.7) is valid and the series in (3.8) and (3.9) converge absolutely. 
PRoof. The Cauchy inequality together with the orthogonality property (3.4) give the estimate

$$
\left|\tilde{a}_{n}\right| \leq \frac{\pi}{\mathbf{K} k^{2 n}} \sum_{m=0}^{\infty} \frac{\left|a_{m}\right|\left|\alpha_{m n}\right|}{\varepsilon_{m} \cosh (\lambda m)} \leq \frac{1}{2 k^{n}} \sqrt{\frac{\pi}{\mathbf{K}}}\left[\sum_{m=0}^{\infty} \frac{\left|a_{m}\right|^{2}}{\cosh (\lambda m)}+1\right] .
$$

Thus the series (3.8) is absolutely convergent and

$$
\left|\tilde{a}_{n}\right| \leq A k^{-n}, \quad A=\text { const. }
$$

Then the series in the right-hand side of (3.7) converges absolutely as well. We substitute (3.8) into the series (3.7). The arising repeated series converges absolutely in virtue of the estimates (3.17) and (3.18). We can interchange the order of summation and on account of the orthogonality relation prove the theorem.

REMARK 3.6. The expansion (3.7) is not unique because there is the absolutely convergent null series

$$
\sum_{n=0}^{\infty} \frac{(2 n-1) ! !}{(2 n) ! !} \alpha_{m n}=0
$$

obtained by setting $x=2 \mathbf{K}$ into the series representation (2.1).

Now consider the sets of the numbers

$$
\alpha_{m n}^{(N)}=\frac{2 \mathbf{K} k^{2 n} \cosh (m \lambda)}{\pi N} \sum_{s=0}^{N-1} \operatorname{dn} \frac{\mathbf{K} s}{N}\left(\operatorname{sn} \frac{\mathbf{K} s}{N}\right)^{2 n} \cos \frac{\pi m s}{N}, \quad N \geq 2,
$$

which are biorthogonal to $\alpha_{\ln }$ with the biorthogonality property

$$
\frac{\pi}{\mathbf{K} \cosh (\lambda m)} \sum_{n=0}^{\infty} \frac{\alpha_{m n} \alpha_{l n}^{(N)}}{k^{2 n}}=\varepsilon_{l} \delta_{l m}, \quad 0 \leq m, l \leq N-1
$$

where integers $m$ and $l$ both are either even or odd.

This biorthogonality property has been obtained by setting $t=2 \mathrm{~K} s / N$ into the series (2.5). After multiplying by $\cos (\pi l s / N)$, we have used the sums

$$
\frac{2}{N} \sum_{s=0}^{N-1} \cos \frac{\pi m s}{N} \cos \frac{\pi l s}{N}=\varepsilon_{l} \delta_{l m}, \quad 0 \leq m, l \leq N-1,
$$

(both integers $m$ and $l$ both are either even or odd).

One might interpret $\alpha_{m n}^{(N)}$ as quadrature formulas for the integral representation of the coefficients $\alpha_{m n}$. Therefore, the coefficients $\alpha_{m n}$ are the limits of 
the numbers $\alpha_{m n}^{(N)}$ as $N \rightarrow \infty$. To estimate the rapidity of convergence of these quadrature formulas, we will expand $\alpha_{m n}^{(N)}$ into the series with respect to $\alpha_{m n}$. This objective can be achieved by employing the finite Poisson formula

$$
\sum_{s=0}^{N} f(\varpi s)=\frac{f(\varpi N)+f(0)}{2}+\frac{1}{\varpi} \sum_{s=0}^{\infty} \varepsilon_{s} \int_{0}^{\varpi N} f(t) \cos \left(\frac{2 \pi s t}{\varpi}\right) d t .
$$

As

$$
f(t)=\frac{2 \mathbf{K} k^{2 n}}{\pi N} \operatorname{dn} \frac{\mathbf{t}}{2}\left(\operatorname{sn} \frac{\mathbf{t}}{2}\right)^{2 n} \cos \frac{\pi m t}{2 \mathbf{K}}, \quad \varpi=\frac{2 \mathbf{K}}{N},
$$

this becomes

$$
\begin{aligned}
\frac{\alpha_{m n}-\alpha_{m n}^{(N)}}{\cosh (m \lambda)}= & \frac{k^{\prime} k^{2 n}(-1)^{m} \mathbf{K}}{\pi N}-\sum_{s=1}^{\infty} \frac{\alpha_{2 s N+m, n}}{\cosh [(2 s N+m) \lambda]} \\
& -\sum_{s=1}^{\infty} \frac{\alpha_{2 s N-m, n}}{\cosh [(2 s N-m) \lambda]} .
\end{aligned}
$$

The series in this relation have fast decreasing terms and wide lacunas. These series decay rapidly as $N \rightarrow \infty$. Then the first term in the right-hand side is a leading term as $(2 N-m) \lambda \gg 1$.

Similar results can be pointed out for $\beta_{m n}, \gamma_{m n}$, and $\zeta_{m n}$ as well. The immediate application is that sums of the series $\sum_{m=0}^{\infty}( \pm 1)^{m} \omega_{m} Q(m) P n_{m}(x)$ are evaluated exactly when $Q(m)$ is an even polynomial in $m$ and $\omega_{m}=$ $\left[\varepsilon_{m} \cosh (\lambda m)\right]^{-1}$. These sums are polynomials in the Jacobian elliptic functions. The same conclusion takes place for analogous Fourier series which may also be useful in applications.

4. Asymptotic expansions. We are interested in studying $P n_{m}(x)$ as $m \rightarrow$ $\infty$. In order to determine an asymptotic expansion, we write

$$
\begin{aligned}
\frac{1}{2} \operatorname{cn} \frac{\sqrt{s}}{2} & \sqrt{\frac{x^{2}-s}{\operatorname{cn}^{2}(\sqrt{s} / 2)-\mathrm{cn}^{2}(x / 2)}} \\
& =\sum_{n=0}^{N-1} a_{n}(x)\left(s-x^{2}\right)^{n}+R(x, s), \quad s=t^{2},
\end{aligned}
$$

where $a_{n}(x)$ are coefficients of the Taylor series about the point $s=x$, and we substitute this expression into the integral representation (1.1). Integration 
yields

$$
\begin{aligned}
P n_{m}(x) & =\sum_{n=0}^{N-1} \tilde{a}_{n}(x)\left(\frac{x}{\mu_{m}}\right)^{n} J_{n}\left(\mu_{m} x\right)+o\left(\frac{1}{\mu_{m}^{N}}\right), \\
\tilde{a}_{n}(x) & =(-1)^{n}(2 n-1) ! ! \pi a_{n}(x), \\
a_{0}(x) & =\sqrt{\frac{x \operatorname{cn}(x / 2)}{2 \operatorname{sn}(x / 2) \operatorname{dn}(x / 2)}}, \\
a_{1}(x) & =\frac{\operatorname{cn}(x / 2)(2 \operatorname{sn}(x / 2) \operatorname{cn}(x / 2) \operatorname{dn}(x / 2)+x v(x))}{8(2 x \operatorname{sn}(x / 2) \operatorname{cn}(x / 2) \operatorname{dn}(x / 2))^{3 / 2}}, \\
v(x) & =k^{2} \operatorname{sn}^{4} \frac{x}{2}-2\left(1-k^{2}\right) \operatorname{sn}^{2} \frac{x}{2}-1 .
\end{aligned}
$$

This asymptotic formula involves the Bessel functions of the first kind $J_{n}(x)$ and is valid for $0 \leq x \leq 2 \mathbf{K}-\varepsilon, \varepsilon>0$. The order of the remainder arises here after $N$ integrations by parts of integrals of the form

$$
\int_{0}^{x}\left(x^{2}-t^{2}\right)^{N-(1 / 2)} \psi(t) \cos \left(\mu_{m} t\right) d t
$$

where $\psi(t) \in C^{\infty}[0,2 \mathbf{K}]$ is an even function, by referring to the well-known Riemann lemma about Fourier coefficients of an absolutely integrable function. As $\mu_{m} x \gg 1$, the truncated error is $O\left(\mu_{m}^{-N-(1 / 2)}\right)$. This follows from the estimate of the Bessel functions.

An asymptotic expansion, which is uniform with respect to the argument of the function on $[\varepsilon, 2 \mathbf{K}]$, can be derived in an analogous manner from (1.9). We utilize the Taylor series about the point $s=x$ :

$$
\frac{1}{2 \sqrt{s}} \operatorname{sn} \frac{\sqrt{s}}{2} \sqrt{\frac{x^{2}-s}{\operatorname{cn}^{2}(\sqrt{s} / 2)-\operatorname{cn}^{2}(x / 2)}}=\sum_{n=0}^{N-1} b_{n}(x)\left(s-x^{2}\right)^{n}+R(x, s), \quad s=t^{2},
$$

and find

$$
\begin{gathered}
P n_{m}(2 \mathbf{K}-x)=(-1)^{m} \sum_{n=0}^{N-1} \tilde{b}_{n}(x)\left(\frac{x}{\mu_{m}}\right)^{n} J_{n+1}\left(\mu_{m} x\right)+o\left(\frac{1}{\mu_{m}^{N}}\right), \\
x \in[0,2 \mathbf{K}-\varepsilon], \tilde{b}_{n}(x)=\pi(-1)^{n+1}(2 n-1) ! ! x b_{n}(x) \operatorname{dn} \frac{x}{2} \\
b_{1}(x)=\frac{\operatorname{sn}(x / 2)}{8 x^{5 / 2}(2 \operatorname{sn}(x / 2) \operatorname{cn}(x / 2) \operatorname{dn}(x / 2))^{3 / 2}}, \quad \operatorname{sd} x=\frac{\operatorname{sn} x}{\operatorname{dn} x}, \\
\times\left[x\left(\operatorname{dn}^{2}\left(\frac{x}{2}\right)+\operatorname{dn}^{2} \operatorname{cn}^{2}\left(\frac{x}{2}\right)+\operatorname{cn}^{2}\left(\frac{x}{2}\right)\right)-6 \operatorname{sn}\left(\frac{x}{2}\right) \operatorname{cn}\left(\frac{x}{2}\right) \operatorname{dn}\left(\frac{x}{2}\right)\right] .
\end{gathered}
$$


The more convenient formula, that holds validity for $\varepsilon \leq x \leq 2 \mathrm{~K}-\varepsilon$, is established as a result of inserting the asymptotic expansion of the Bessel functions into (4.2):

$$
\begin{aligned}
P n_{m}(x) & =\frac{\cos \left(\mu_{m} x-(\pi / 4)\right)}{\sqrt{\mu_{m} p(x)}}-\frac{r(x)}{\mu_{m}^{3 / 2}} \sin \left(\mu_{m} x-\frac{\pi}{4}\right)+O\left(\frac{1}{\mu_{m}^{5 / 2}}\right) \\
p(x) & =\frac{\operatorname{sn}(x / 2)}{\pi \operatorname{cd}(x / 2)}, \quad r(x)=\frac{v(x) \sqrt{\pi}}{16(\operatorname{sn}(x / 2) \operatorname{dn}(x / 2))^{3 / 2} \sqrt{\operatorname{cn}(x / 2)}} .
\end{aligned}
$$

Further we will need an asymptotic expansion which is uniform on $[0,2 \mathbf{K}]$. This can be derived by employing the following Taylor series about the point $s=\sin ^{2}(\theta / 2), \theta=\pi x / 2 \mathbf{K}$ :

$$
\begin{gathered}
\frac{\operatorname{cn}(\rho(s))}{\sqrt{1-s}} \sqrt{\frac{\sin ^{2}(\theta / 2)-s}{\operatorname{cn}^{2}(\rho(s))-\operatorname{cn}^{2}(x / 2)}}=\sum_{n=0}^{N} c_{n}(x)\left(s-\sin ^{2} \frac{\theta}{2}\right)^{n}+R(x, s), \\
\rho(s)=\frac{2 \mathbf{K}}{\pi} \arcsin \sqrt{s}, \quad s=\sin ^{2} \frac{\pi t}{4 \mathbf{K}} .
\end{gathered}
$$

This leads to the final result of the section

$$
\begin{aligned}
P n_{m}(x) & =\sum_{n=0}^{N-1} \tilde{c}_{n}(x) \frac{(m-n-1) !}{(m-1) !} \sin ^{2 n} \theta P_{m-n-1}^{(n, n+1)}(\cos \theta)+O\left(\frac{1}{\mu_{m}^{N+1 / 2}}\right) \\
\tilde{c}_{n}(x) & =(-1)^{n} 2^{1-3 n}(2 n-1) ! ! K c_{n}(x) \cos ^{2} \frac{\theta}{2} \\
c_{0}(x) & =\sqrt{\frac{\pi \operatorname{cd}(x / 2) \tan (\theta / 2)}{2 \mathbf{K} \operatorname{sn}(x / 2)}}
\end{aligned}
$$

The integral

$$
\begin{aligned}
\mathscr{P}_{n, m}(\theta) & =\int_{0}^{\theta} \frac{\cos (t / 2) \cos m t d t}{\left(\cos ^{2}(t / 2)-\cos ^{2}(\theta / 2)\right)^{1 / 2-n}} \\
& =\frac{\pi(2 n-1) ! !}{2^{2 n+1}} \sin ^{n} \theta\left[P_{m}^{-n}(\cos \theta)+P_{m-1}^{-n}(\cos \theta)\right]
\end{aligned}
$$

was utilized in the evaluation. After that, associated Legendre functions were expressed in terms of Jacobi polynomials $P_{m}^{(\alpha, \beta)}(x)$ by means of the relations [5]

$$
\begin{gathered}
P_{m}^{-n}(x)=\left(1-x^{2}\right)^{n / 2} \frac{\Gamma(m-n+1)}{\Gamma(m+n+1)} \frac{d^{n} P_{m}^{(0,0)}(x)}{d x^{n}}, \\
\frac{d^{n} P_{m}^{(0,0)}(x)}{d x^{n}}=\frac{\Gamma(m+n+1)}{2^{n} \Gamma(m+1)} P_{m-n}^{(n, n)}(x) \\
(1+x) P_{m-1}^{(n, n+1)}(x)=\frac{m}{(m+n)} P_{m}^{(n, n)}(x)+P_{m-1}^{(n, n)}(x) .
\end{gathered}
$$


The order of the truncated error has been found by integrating by parts and using the uniform weight estimate of the Jacobi polynomial

$$
\left(\sin \frac{\theta}{2}\right)^{\alpha+1 / 2}\left(\cos \frac{\theta}{2}\right)^{\beta+1 / 2}\left|P_{m}^{(\alpha, \beta)}(\cos \theta)\right| \leq \frac{A}{\sqrt{m}}, \quad A=\text { const. }
$$

We note here that $\mathscr{P}_{0, m}(\theta)=\pi \cos ^{2}(\theta / 2) P_{m-1}^{(0,1)}(\cos \theta)$ is the limit of $P n_{m}(x)$ as $k \rightarrow 0$.

5. Expanding functions in the $P n_{m}(x)$ and $\widetilde{P} n_{m}(x)$ series. The limiting functions $\mathscr{P}_{0, m}(\theta)$ are orthogonal. One might expect $P n_{m}(x)$ to be orthogonal as well. Our starting point is the observation that the integral representation (1.10) is the solution of the Abel integral equation

$$
\int_{0}^{t} \frac{m \operatorname{sn}(x / 2)}{2 \operatorname{Kcd}(x / 2)} P n_{m}(x) \frac{d x}{\sqrt{\operatorname{cn}^{2}(x / 2)-\mathrm{cn}^{2}(t / 2)}}=\frac{\sin \left(\mu_{m} t\right)}{\operatorname{cn}(t / 2)} .
$$

We multiply this relation by $(1 / \mathbf{K}) \mathrm{cn}(t / 2) \sin (\pi m t / 2 \mathbf{K})$ and integrate with respect to $t$ between 0 and $2 \mathbf{K}$. A change of the order of integration coupled with (1.7) gives

$$
\frac{m \tanh (\lambda m)}{2 \mathbf{K}^{2}} \int_{0}^{2 \mathbf{K}} \frac{\operatorname{sn}(x / 2)}{\operatorname{cd}(x / 2)} P n_{m}(x) P n_{l}(x) d x=\delta_{m l},
$$

where $\delta_{m l}$ is the Kronecker delta.

THEOREM 5.1. The set of the functions

$$
\mathfrak{H} \mathfrak{n}_{m}(x)=\sqrt{\frac{m \tanh (\lambda m) \operatorname{sn}(x / 2)}{2 \mathbf{K}^{2} \operatorname{cd}(x / 2)}} P n_{m}(x), \quad m=1,2, \ldots,
$$

is an orthonormal basis in $L_{2}[0,2 \mathrm{~K}]$. In other words, for an arbitrary function $f(x) \in L_{2}[0,2 \mathbf{K}]:$

$$
\lim _{M \rightarrow \infty}\left\|f(x)-\sum_{m=1}^{M}\left(f, \mathfrak{P} \mathfrak{n}_{m}\right) \mathfrak{P O} \mathfrak{n}_{m}(x)\right\|=0 .
$$

Moreover, the Parseval formula

$$
\|f(x)\|^{2}=\sum_{m=1}^{\infty}\left(f, \not \mathfrak{O n} n_{m}\right)^{2}
$$

is valid.

Proof. According to the uniform asymptotic expansion (4.8), as $m \rightarrow \infty$,

$$
\mathfrak{J}_{m}=\left\|\not \mathfrak{O} \mathfrak{n}_{m}(x)-\sqrt{\frac{m \pi}{2 \mathbf{K}} \sin \left(\frac{\pi x}{2 \mathbf{K}}\right)} P_{m-1}^{(0,1)}\left(\cos \frac{\pi x}{2 \mathbf{K}}\right) \cos \left(\frac{\pi x}{4 \mathbf{K}}\right)\right\|^{2}=O\left(\frac{1}{m^{2}}\right) .
$$


This estimate shows that the series $\sum_{m=1}^{\infty} \mathfrak{J}_{m}$ is convergent. Since the set of the functions $\sqrt{(m \pi / 2 \mathbf{K}) \sin (\pi x / 2 \mathbf{K})} P_{m-1}^{(0,1)}(\cos (\pi x / 2 \mathbf{K})) \cos (\pi x / 4 \mathbf{K})$ (the Jacobi polynomials) is an orthonormal basis in $L_{2}[0,2 \mathbf{K}]$ and the functions $\not \mathfrak{O n} \mathfrak{n}_{m}(x)$ constitute an orthonormal set, the theorem is the immediate corollary of the results about disturbed complete orthonormal sets [2, 6].

Pointwise convergence of the series requires introducing a different class of functions.

THEOREM 5.2. Suppose $f(x) \in L_{1}[0,2 \mathbf{K}]$. Then the Abel means

$$
\tilde{f}_{\tau}(x)=\sum_{m=1}^{\infty}\left(f, \mathfrak{P} \mathfrak{n}_{m}\right) \mathfrak{P} \mathfrak{\bullet} \mathfrak{n}_{m}(x) \exp (-\tau m)
$$

converges to $f(x),\left(\tilde{f}_{\tau}(x) \rightarrow f(x)\right.$ as $\left.\tau \rightarrow+0\right)$, at every point $x \in(0,2 \mathbf{K})$, where $f(x)$ is the derivative of its integral.

Proof. We write

$$
\begin{aligned}
\tilde{f}_{\tau}(x) & =\frac{1}{\mathbf{K}} \sum_{m=1}^{\infty} \exp [-\tau m]\left(f, \sin \left(\mu_{m} s\right)\right) \sin \left(\mu_{m} t\right)+\int_{0}^{2 \mathbf{K}} f(s) F_{\tau}(x, s) d s, \\
F_{\tau}(x, s) & =\sum_{m=1}^{\infty} \exp [-\tau m]\left[\mathfrak{P} \mathfrak{n}_{m}(s) \not \mathfrak{O n} \mathfrak{n}_{m}(x)-\frac{1}{\mathbf{K}} \sin \left(\mu_{m} s\right) \sin \left(\mu_{m} x\right)\right] .
\end{aligned}
$$

In order to prove our theorem, we introduce three functions which are analytical in the upper half-plane $\mathfrak{k e}(z)>0$ and real on the imaginary axis:

$$
\begin{aligned}
P(z, x) & =\int_{0}^{x} \frac{\operatorname{cn}(t / 2) \cos (\pi z t / 2 \mathbf{K})}{\sqrt{\mathrm{cn}^{2}(t / 2)-\mathrm{cn}^{2}(x / 2)}} d t \\
Q^{ \pm}(z, s) & =\int_{s}^{2 \mathbf{K}} \frac{\operatorname{cn}(t / 2) \exp [ \pm i \pi z t / 2 \mathbf{K}]}{\sqrt{\mathrm{cn}^{2}(s / 2)-\mathrm{cn}^{2}(t / 2)}} d t \\
& =\frac{\operatorname{dn}((2 \mathbf{K}-s) / 2)}{\exp (\mp i \pi z)} \int_{0}^{2 \mathbf{K}-s} \frac{\operatorname{sn}(t / 2) \exp [\mp i \pi z t / 2 \mathbf{K}]}{\sqrt{\operatorname{sn}^{2}(\mathbf{K}-s / 2)-\operatorname{sn}^{2}(t / 2)}} d t .
\end{aligned}
$$

Their asymptotic behavior as $z \rightarrow \infty$ and $\mathfrak{k e}(z) \geq 0$ can be elucidated by utilizing the Taylor series in (4.1) and (4.4), the integral representations of the Bessel and Struve functions as well as the asymptotic expansion of the Struve function [5]

$$
\begin{aligned}
& P(z, x) \sim \sum_{n=0}^{2} \tilde{a}_{n}(x)\left(\frac{x}{\pi z}\right)^{n} J_{n}\left(\frac{\pi z x}{2 \mathbf{K}}\right)+O\left(|z|^{-3}\right) \exp \left[-\frac{i z \pi x}{2 \mathbf{K}}\right], \quad x<2 \mathbf{K}-\varepsilon, \\
& Q^{ \pm}(z, s)= \pm \exp [ \pm i z \pi] \sum_{n=0}^{1} \frac{g_{n}(s)}{z^{n}} H_{n+1}^{ \pm}\left(\pi z\left(1-\frac{s}{2 \mathbf{K}}\right)\right)+R^{ \pm}(z), \quad \varepsilon<s \leq 2 \mathbf{K},
\end{aligned}
$$


where $g_{n}(s)=i \tilde{b}_{n}(2 \mathbf{K}-s)(2 \mathbf{K}(2 \mathbf{K}-s) / \pi)^{n}$; coefficients $\tilde{a}_{n}(x)$ and $\tilde{b}_{n}(x)$ are defined as in (4.2) and (4.5), respectively; $H_{n}^{-}(x)=H_{n}^{(1)}(x), H_{n}^{+}(x)=H_{n}^{(2)}(x)$, and $H_{n}^{(r)}(x)$ are the Bessel functions of the third kind; and

$$
\begin{aligned}
& R^{+}(z)=O\left(|z|^{-2}\right) \exp \left(\frac{i \pi z s}{2 \mathbf{K}}\right) \\
& R^{-}(z)=O\left(|z|^{-2}\right) \exp (i z \pi)
\end{aligned}
$$

Consider the integral taken along a closed contour

$$
\oint_{\mathscr{L}_{N}} \exp [-\tau z] \frac{\exp [i \pi z]}{\sin \pi z}\left[\tilde{Q}(z, s) P(z, x)-\sin \left(\frac{\pi z s}{2 \mathbf{K}}\right) \sin \left(\frac{\pi z x}{2 \mathbf{K}}\right)\right] d z
$$

where $s \geq x>0$,

$$
\begin{aligned}
& \tilde{Q}(z, s)=\mathbf{K} z \rho(s, x) \frac{Q^{+}(z, s)-Q^{-}(z, s)}{2 i}, \\
& \rho(s, x)=\frac{1}{2 \mathbf{K}^{2}} \sqrt{\frac{\operatorname{sn}(x / 2) \operatorname{sn}(s / 2)}{\operatorname{cd}(x / 2) \operatorname{cd}(s / 2)}} .
\end{aligned}
$$

The contour $\mathscr{L}_{N}$ consists of the $\operatorname{arc}|z|=N+1 / 2,0 \leq \arg z \leq \pi / 2$; the arcs of the small radius $\varepsilon$ with centers $z=1,2, \ldots, N$, which lie within the first quadrant; and the segments of the real and imaginary axes joining the ends of the arcs. The integrand is an analytic function in the domain bounded by $\mathscr{L}_{N}$ and hence the integral is zero. As $\varepsilon \rightarrow 0, N \rightarrow \infty$, the imaginary part of the integral tends to $F_{T}(x, s)$ and some integrals. This yields

$$
\begin{gathered}
F_{\tau}(x, s)=\int_{0}^{\infty} \sin \tau z \frac{\exp [-\pi z]}{\pi \mathbf{K} \sinh (\pi z)}\left[\widetilde{Q}(i z, s) P(i z, x)+\sinh \frac{\pi z s}{2 \mathbf{K}} \sinh \frac{\pi z x}{2 \mathbf{K}}\right] d z \\
+\int_{0}^{\infty} \frac{\exp [-\tau z]}{\pi}\left[z \rho(s, x) P(z, x) \mathfrak{I m} Q^{+}(z, s)\right. \\
\left.\quad-\frac{1}{\mathbf{K}} \sin \frac{\pi z s}{2 \mathbf{K}} \sin \frac{\pi z x}{2 \mathbf{K}}\right] d z .
\end{gathered}
$$

Similarly, the second integral in (5.14), which is denoted further by $\widetilde{F}_{\tau}(x, s)$, can be transformed to the nonoscillating form by examining the contour integral

$$
\oint_{\mathscr{L}} \exp [-\tau z]\left[z \rho(s, x) Q^{+}(z, s) P(z, x)-\frac{1}{\mathbf{K}} \exp \left(\frac{i \pi z s}{2 \mathbf{K}}\right) \sin \left(\frac{\pi z x}{2 \mathbf{K}}\right)\right] d z=0, \quad s \geq x .
$$

The closed integration path $\mathscr{L}$ consists of the $\operatorname{arc}|z|=N, 0 \leq \arg z \leq \pi / 2$, and the segments of the real and imaginary axes which emanate from the origin. 
We have

$$
\begin{aligned}
\widetilde{F}_{T}(x, s)=\frac{1}{\pi} \int_{0}^{\infty} \sin \tau z[ & z \rho(s, x) P(i z, x) Q^{+}(i z, s) \\
& \left.-\frac{1}{\mathbf{K}} \exp \left[-\frac{\pi z s}{2 \mathbf{K}}\right] \sinh \frac{\pi z x}{2 \mathbf{K}}\right] d z
\end{aligned}
$$

in the limit $N \rightarrow \infty$. Thus the kernel $F_{T}(x, s)$ is the Fourier sine transform of a certain function (if $s<x$, then $F_{T}(x, s)=F_{T}(s, x)$ ). It follows from the asymptotic expansions of $Q^{ \pm}(z, s)$ and $P(z, x)$ that the integrand $q(z, x, s, \tau)$ of this Fourier transform has the structure

$$
q(z, x, s, \tau)= \begin{cases}q_{1}(z, x, s)+\frac{1}{z} F(x, s) \exp \left[-\frac{\pi}{2 \mathbf{K}} z|s-x|\right] \sin \tau z, & z \geq 1 \\ q_{1}(z, x, s) \sin \tau z, & 0 \leq z<1\end{cases}
$$

where $F(x, s)$ is a bounded function, $q_{1}(z, x, s)$ is an absolutely integrable function of $z$ for $s \in[0,2 \mathbf{K}]$ and $x \in(0,2 \mathbf{K})$. Then

$F_{\tau}(x, s)=F(x, s)\left[\arctan \frac{2 \mathbf{K} \tau}{\pi|s-x|}-\int_{0}^{\tau} \frac{\sin z}{z} \exp \left[-\frac{z \pi|s-x|}{2 \mathbf{K} \tau}\right] d z\right]+G_{\tau}(x, s)$,

where a bounded function $G_{\tau}(x, s)$ is a Fourier sine integral which converges uniformly with respect to $\tau$. Hence, $F_{\tau}(x, s)$ is a bounded function as well and $F_{\tau}(x, s) \rightarrow 0$ as $\tau \rightarrow+0, s \neq x$. Now we see that the integral in (5.8) vanishes as $\tau \rightarrow+0$ and the summation of the Abel means that $\tilde{f}_{\tau}(x)$ is reduced to Abel summation of the Fourier series. In correspondence to Fatou's theorem [4], our theorem is proved.

The requirement of the Abel summation is essential in the proven theorem because there is an absolutely integrable function whose $P n_{m}(x)$-development diverges almost everywhere. That can be proved exactly in the same way as for Legendre polynomials [1]. The next theorem shows that this situation changes for the better when some extra restrictions on behavior of a function are imposed.

THEOREM 5.3. Suppose $\sqrt{x /(2 \mathbf{K}-x)} f(x)$ is an absolutely integrable function over the domain $[0,2 \mathbf{K}]$. If $f(x)$ is of bounded variation in the vicinity of the point $s \in(0,2 \mathbf{K})$, then

$$
\begin{gathered}
\frac{f(s+0)+f(s-0)}{2}=\sum_{m=1}^{\infty} m f_{m} \tanh (\lambda m) P n_{m}(s), \\
f_{m}=\frac{1}{2 \mathbf{K}^{2}} \int_{0}^{2 \mathbf{K}} \frac{\operatorname{sn}(x / 2)}{\operatorname{cd}(x / 2)} f(x) P n_{m}(x) d x .
\end{gathered}
$$


Proof. Decompose $f(x)$ into the sum of two truncated functions:

$$
g(x)=\left\{\begin{array}{ll}
f(x), & x \leq x_{0}, \\
0, & x<x_{0},
\end{array} \quad h(x)= \begin{cases}0, & x<x_{0}, \\
f(x), & x>x_{0},\end{cases}\right.
$$

where $x_{0}>s$. We will show that the theorem is valid for each of these functions.

Examine the loop integral

$$
\begin{gathered}
\oint_{\mathscr{L}_{N}^{*}} z \Psi(z, s, x) d z=0, \quad 0 \leq x \leq x_{0}<2 \mathbf{K}, \\
\Psi(s, x, s)=\frac{\exp [i \pi z]}{z \sin (\pi z)} \widetilde{Q}(z, s) P(z, x)-i \frac{\pi^{2} \sqrt{x s} H_{0}^{(!)}(\pi z)}{4 \mathbf{K}^{2} J_{0}(\pi z)} J_{0}\left(\frac{\pi z s}{2 \mathbf{K}}\right) J_{0}\left(\frac{\pi z x}{2 \mathbf{K}}\right),
\end{gathered}
$$

where $N \gg 1$ and the contour $\mathscr{L}_{N}^{*}$ consists of the $\operatorname{arc}|z|=N+1 / 2,0 \leq$ $\arg z \leq \pi / 2$; the arcs of the small radius $\varepsilon$ with centers $z=n, z=\gamma_{n} / \pi$ ( $n=1,2, \ldots, N)$, which lie within the first quadrant; and the segments of the real and imaginary axes joining the ends of the arcs. Here $\gamma_{n}$ are zeros of the Bessel function $J_{0}\left(\gamma_{n}\right)=0$. These zeros have asymptotic expansions $\gamma_{n}=(n-$ $1 / 4) \pi+O(1 / n)$ as $n \gg 1[10]$. We also use the notations from Theorem 5.2.

In the manner of Theorem 5.2, upon utilizing the Wronskian of the Bessel functions

$$
H_{1}^{(!)}(z) J_{0}(z)-H_{0}^{(!)}(z) J_{1}(z)=\frac{2}{\pi i z}
$$

we achieve, as $\varepsilon \rightarrow 0$, the formula

$$
\begin{aligned}
\sum_{m=1}^{N}\left[\rho(s, x) m \tanh \lambda m P n_{m}(s) P n_{m}(x)\right. & \\
& \left.-\frac{\sqrt{x s} J_{0}\left(\pi \gamma_{m} s / 2 \mathbf{K}\right) J_{0}\left(\pi \gamma_{m} x / 2 \mathbf{K}\right)}{2 \mathbf{K}^{2} J_{1}^{2}\left(\gamma_{m}\right)}\right]=T_{N}(s, x) .
\end{aligned}
$$

Here $T(s, x)$ is defined as

$$
\begin{aligned}
& T_{N}(s, x)=\operatorname{Re} \int_{0}^{\pi / 2} z^{2}[\Psi(z, x, s)+V(z, x, s)] d \theta, \quad z=\left(N+\frac{1}{2}\right) \exp (i \theta), \\
& V(z, x, s)= \begin{cases}\rho(s, x) Q^{+}(z, s) P(z, x)-i \frac{\pi^{2} \sqrt{x s}}{4 \mathbf{K}^{2}} H_{0}^{(1)}\left(\frac{\pi z s}{2 \mathbf{K}}\right) J_{0}\left(\frac{\pi z x}{2 \mathbf{K}}\right), \quad s \geq x, \\
\rho(s, x) Q^{+}(z, x) P(z, s)-i \frac{\pi^{2} \sqrt{x s}}{4 \mathbf{K}^{2}} H_{0}^{(1)}\left(\frac{\pi z x}{2 \mathbf{K}}\right) J_{0}\left(\frac{\pi z s}{2 \mathbf{K}}\right), \quad x \leq s .\end{cases}
\end{aligned}
$$

According to the uniform asymptotic expansions (5.10), $T_{N}(s, x)$ is a bounded function for $0<s<2 \mathbf{K}, 0 \leq x \leq x_{0}<2 \mathbf{K}, 0<N \leq \infty$, and $T_{N}(s, x) \rightarrow 0$ as 
$N \rightarrow \infty, s \neq x$. Now we can write

$$
\begin{aligned}
\lim _{N \rightarrow \infty} \sum_{m=1}^{N} m g_{m} \tanh (\lambda m) P n_{m}(s) & \\
= & \lim _{N \rightarrow \infty} \int_{0}^{x_{0}} \sqrt{\frac{\operatorname{sn}(x / 2) \operatorname{cd}(s / 2)}{\operatorname{cd}(x / 2) \operatorname{sn}(s / 2)}} g(x) T_{N}(s, x) d x \\
+ & \lim _{N \rightarrow \infty} \frac{\pi^{2}}{4 \mathbf{K}^{2}} \sum_{m=1}^{N} J_{1}^{-2}\left(\gamma_{n}\right) J_{0}\left(\frac{\pi \gamma_{n} s}{2 \mathbf{K}}\right) \int_{0}^{x_{0}} \sqrt{\frac{s x \operatorname{sn}(x / 2) \operatorname{cd}(s / 2)}{\operatorname{cd}(x / 2) \operatorname{sn}(s / 2)}} \\
& \times g(x) J_{0}\left(\frac{\pi \gamma_{n} x}{2 \mathbf{K}}\right) d x .
\end{aligned}
$$

The first integral in the right-hand side converges uniformly with respect to $N$ and vanishes as $N \rightarrow \infty$. Since the second term in the right-hand side is the Fourier-Bessel series expansion of $\sqrt{\operatorname{sn}(x / 2) / x \operatorname{cd}(x / 2)} g(x)$ [10], we obtain

$$
\sum_{m=1}^{\infty} m g_{m} \tanh (\lambda m) P n_{m}(s)=\frac{f(s+0)+f(s-0)}{2}
$$

In order to prove the theorem for function $h(x)$, we introduce three functions of a complex variable $z$ :

$$
\begin{aligned}
\hat{P}(z, x) & =\int_{0}^{x} \frac{\operatorname{sn}(t / 2) \sin (\pi z t / 2 \mathbf{K})}{\sqrt{\mathrm{cn}^{2}(t / 2)-\mathrm{cn}^{2}(x / 2)}} d t \\
\hat{Q}^{ \pm}(z, s) & =\int_{s}^{2 \mathbf{K}} \frac{\operatorname{sn}(t / 2) \exp [ \pm i \pi z t / 2 \mathbf{K}]}{\sqrt{\mathrm{cn}^{2}(s / 2)-\mathrm{cn}^{2}(t / 2)}} d t, \quad s>0 .
\end{aligned}
$$

Let $u=2 \mathbf{K}-s$. It may be proved in the same manner as above that

$$
\begin{aligned}
& \frac{4 \mathbf{K}^{2}}{\pi^{2}} \sum_{m=1}^{\infty} m h_{m} \tanh (\lambda m) P n_{m}(s) \\
& =\frac{2 \operatorname{dn}(u / 2)}{\pi^{2}} \sum_{m=1}^{\infty} m \tanh (\lambda m) \widetilde{P} n_{m}(u) \int_{0}^{2 \mathbf{K}-x_{0}} \frac{\operatorname{cn}(x / 2)}{\operatorname{sn}(x / 2)} h(2 \mathbf{K}-x) \tilde{P} n_{m}(x) d x \\
& =\sum_{m=1}^{\infty} J_{2}^{-2}\left(\gamma_{n}\right) J_{1}\left(\frac{\pi \gamma_{n} u}{2 \mathbf{K}}\right) \int_{0}^{2 \mathbf{K}-x_{0}} \sqrt{\frac{x u \operatorname{sn}(u / 2) \operatorname{cd}(x / 2)}{\operatorname{cd}(u / 2) \operatorname{sn}(x / 2)}} \\
& \times h(2 \mathbf{K}-x) J_{1}\left(\frac{\pi \gamma_{n} x}{2 \mathbf{K}}\right) d x=0 .
\end{aligned}
$$

Then combining (5.26) and (5.28) yields (5.19).

The preceding results lead to analogous theorems about expanding functions into the $\widetilde{P} n_{m}(x)$-series. It is attained by mere changes of the variables. 
THEOREM 5.4. The set of the functions

$$
\tilde{\mathcal{P}} \mathfrak{n}_{m}(x)=\sqrt{\frac{m \tanh (\lambda m) \operatorname{cn}(x / 2)}{2 \mathbf{K}^{2} \operatorname{sd}(x / 2)}} \widetilde{P} n_{m}(x), \quad m=1,2, \ldots
$$

is an orthonormal basis in $L_{2}[0,2 \mathrm{~K}]$. Abel summation provides pointwise convergence of the corresponding expansion for any function $f(x) \in L_{1}[0,2 \mathbf{K}]$ :

$$
\lim _{\tau \rightarrow+0} \sum_{m=1}^{\infty} \exp [-\tau m]\left(f, \tilde{\mathcal{P}} \mathfrak{n}_{m}\right) \tilde{\mathcal{P}} \mathfrak{n}_{m}(x)=f(x),
$$

at every point $t=x$ where $f(t)$ is the derivative of its integral.

THEOREM 5.5. Suppose $\sqrt{(2 \mathbf{K}-x) / x} f(x)$ is an absolutely integrable function over the domain $[0,2 \mathbf{K}]$. If $f(x)$ is of bounded variation in the vicinity of the point $s \in(0,2 \mathbf{K})$, then

$$
\begin{gathered}
\frac{f(s+0)+f(s-0)}{2}=\sum_{m=1}^{\infty} m \tilde{f}_{m} \tanh (\lambda m) \widetilde{P} n_{m}(s), \\
\tilde{f}_{m}=\frac{1}{2 \mathbf{K}^{2}} \int_{0}^{2 \mathbf{K}} \frac{\operatorname{cn}(x / 2)}{\operatorname{sd}(x / 2)} f(x) \widetilde{P} n_{m}(x) d x .
\end{gathered}
$$

Evaluate some integrals and series including $P n_{m}(x)$ and $\widetilde{P} n_{m}(x)$. We take into account that the integral representations (1.1) and (1.6) are equivalent to the Fourier series

$$
\begin{aligned}
& \sum_{m=1}^{\infty} P n_{m}(x) \cos \left(\mu_{m} t\right)=-\mathbf{K}\left(k \operatorname{sn} \frac{x}{2}\right)+ \begin{cases}0, & t>x \\
\frac{\mathbf{K c n}(t / 2)}{\sqrt{\operatorname{sn}^{2}(x / 2)-\operatorname{sn}^{2}(t / 2)}}, & t<x\end{cases} \\
& \sum_{m=1}^{\infty} \frac{P n_{m}(x)}{\cosh (\lambda m)} \cos \left(\mu_{m} t\right)=\frac{\mathbf{K d n}(t / 2)}{\sqrt{1-k^{2} \operatorname{sn}^{2}(x / 2) \operatorname{sn}^{2}(t / 2)}}-\mathbf{K}\left(k \operatorname{sn} \frac{x}{2}\right)
\end{aligned}
$$

Hence, by setting $t=2 \mathbf{K}$ and $t=0$, we derive the expansion for the unit function

$$
\frac{1}{\mathbf{K}} \sum_{m=1}^{\infty}\left[\frac{1}{\cosh (\lambda m)}-(-1)^{m}\right] P n_{m}(x)=1, \quad x \in(0,2 \mathbf{K}) .
$$

This result also might be written by virtue of Theorem 5.3. In this case, direct evaluation of coefficients of the expansion should utilize the integral representation (1.10).

Another series can be derived from (5.33), where $t=0$, and from (3.13):

$$
\frac{1}{\mathbf{K}} \sum_{m=1}^{\infty}\left(\frac{(2 n) ! ! \alpha_{m n}}{k^{2 n}(2 n-1) ! !}-1\right) \frac{P n_{m}(x)}{\cosh (\lambda m)}=\operatorname{sn}^{2 n} \frac{x}{2}-1 .
$$


This absolutely convergent series can be inverted by applying the orthogonality relation (5.2). We achieve the new integral representation for $\alpha_{m r}$ :

$$
\frac{(2 r) ! ! \alpha_{m r}}{k^{2 r}(2 r-1) ! !}=1-\frac{m \sinh (\lambda m)}{2 \mathbf{K}} \int_{0}^{2 \mathbf{K}}\left(1-\operatorname{sn}^{2 r} \frac{x}{2}\right) \frac{\operatorname{sn}(x / 2)}{\operatorname{cd}(x / 2)} P n_{m}(x) d x .
$$

THEOREM 5.6. If $f(x)$ has on $[0,2 \mathbf{K}]$ the absolutely convergent expansion of the form

$$
f(x)=\sum_{r=0}^{\infty} q_{r} x^{2 r}
$$

then

$$
\frac{1}{2 \mathbf{K}} \int_{0}^{2 \mathbf{K}} \frac{\operatorname{sn}(x / 2)}{\operatorname{cd}(x / 2)} f(x) P n_{m}(x) d x=\frac{(-1)^{m} f(2 \mathbf{K})}{m \tanh (\lambda m)}+\frac{1}{m \sinh (\lambda m)} \sum_{r=0}^{\infty} \tilde{q}_{r} \alpha_{m r},
$$

where

$$
\begin{gathered}
\tilde{q}_{r}=\frac{(2 r) ! !}{\mathbf{K} k^{2 r}(2 r-1) ! !} \sum_{n=0}^{r} 2^{2 n} q_{n} b_{r-n, n}, \quad b_{0, n}=1, \\
b_{l, n}=\frac{1}{l} \sum_{s=1}^{l} \frac{(n+1) s-l}{2 l+1} c_{s} b_{l-s, n}, \quad c_{s}=\sum_{j=0}^{s} \frac{(2 j-1) ! !(2 s-2 j-1) ! !}{(2 j) ! !(2 s-2 j) ! !} k^{2 j} .
\end{gathered}
$$

Proof. Our starting point is the expansion

$$
\frac{x}{2}=\int_{0}^{\operatorname{sn}(x / 2)} \frac{d t}{\sqrt{\left(1-t^{2}\right)\left(1-k^{2} t^{2}\right)}}=\operatorname{sn} \frac{x}{2} \sum_{s=0}^{\infty} \frac{c_{s}}{2 s+1} \operatorname{sn}^{2 s} \frac{x}{2}
$$

found by substituting the power series for integrand. This positive series converges for all $x \in[0,2 \mathbf{K}]$ because $c_{s}=O(1 / \sqrt{s})$ as $s \gg 1$. Then we have positive convergent series for the powers of $x$ :

$$
x^{2 n}=2^{2 n} \sum_{r=n}^{\infty} b_{r-n, n} \operatorname{sn}^{2 r} \frac{x}{2}
$$

After inserting (5.41) into (5.37), we are permitted to interchange the order of summation. This yields

$$
f(x)=f(2 \mathbf{K})-\sum_{r=0}^{\infty}\left(\sum_{n=0}^{r} 2^{2 n} q_{n} b_{r-n, n}\right)\left(1-\operatorname{sn}^{2 r} \frac{x}{2}\right) .
$$

Now, upon integrating term by term, we achieve the final formula (5.38).

There is another sort of the recurrent relation for $b_{l, n}$ which is rather simpler than the one pointed out above. We write the power series for $\cos (a x)$ 
and replace $x^{2 n}$ by the expression in (5.41). After interchanging the order of summation, it becomes

$$
\cos (a x)=1+\sum_{n=1}^{\infty} \Pi_{n}(a) \operatorname{sn}^{2 n} \frac{x}{2}, \quad \Pi_{n}(a)=\sum_{s=1}^{n} \frac{(-1)^{s} b_{n-s, s}}{(2 s) !} a^{2 s} .
$$

Hence, the coefficients $\beta_{m n}$ have the "explicit" polynomial representation $\beta_{m n}$ $=-\left(n / 2 \mu_{n}^{2}\right) \Pi_{n}\left(\mu_{n}\right)$. Upon substituting these polynomials in the recurrent relation for $\beta_{m n}$, we find

$$
\begin{aligned}
& n(2 n-1) b_{n-s, s}-2\left(1+k^{2}\right)(n-1)^{2} b_{n-1-s, s}+(2 n-3)(n-2) k^{2} b_{n-2-s, s} \\
& \quad=4 s(2 s-1) b_{n-s, s-1}, \quad 1 \leq s<n-2 .
\end{aligned}
$$

The reciprocal expansions for $P n_{l}(x)$ and $\widetilde{P} n_{m}(x)$ follow from (5.33). We write

$$
\sum_{m=1}^{\infty} \frac{P n_{m}(x)}{\cosh (\lambda m)}\left[\cos \frac{\pi m t}{2 \mathbf{K}}-(-1)^{m}\right]=\frac{\mathbf{K d n}(t / 2)}{\sqrt{1-k^{2} \operatorname{sn}^{2}(x / 2) \operatorname{sn}^{2}(t / 2)}}-\frac{\mathbf{K} k^{\prime}}{\operatorname{dn}(x / 2)}
$$

Now multiply (5.45) by $(\operatorname{dn}(t / 2))^{-1} \cos (\pi l t / 2 \mathbf{K})$ and integrate with respect to $t$ from 0 to $2 \mathbf{K}$. Then (1.8) together with the Fourier coefficients of $(\operatorname{dn}(t / 2))^{-1}$ (see [5]) and (5.34) yield

$$
\begin{aligned}
& (-1)^{l} \widetilde{P} n_{l}(x) \\
& \quad=\pi \sum_{m=1}^{\infty} \frac{(-1)^{m} P n_{m}(x)}{\mathbf{K} k^{\prime} \cosh \lambda m}\left[\frac{2 \cosh (\lambda m) \cosh ^{2}(\lambda l)}{\cosh (2 \lambda m)+\cosh (2 \lambda l)}-1\right]+\frac{\pi}{\operatorname{dn}(x / 2)} .
\end{aligned}
$$

The $P n_{m}(x)$-series for $\pi / \operatorname{dn}(x / 2)$ may be evaluated by setting $t=0$ into (5.45). Finally, the relation (5.46) takes the form

$$
(-1)^{l} \sqrt{k^{\prime}} \widetilde{P} n_{l}(x)=\sum_{m=1}^{\infty} P n_{m}(x) f_{m l}
$$

where the numbers $f_{m l}$ are defined as

$$
f_{m l}=\frac{2 \pi(-1)^{m+1} \sinh ^{2}(\lambda m)}{\mathbf{K} \sqrt{k^{\prime}}[\cosh 2 \lambda m+\cosh 2 \lambda l]} .
$$

By changing from $x$ to $2 \mathbf{K}-x$, we get

$$
P n_{l}(x)=\sum_{m=1}^{\infty}(-1)^{m} \sqrt{k^{\prime}} \widetilde{P} n_{m}(x) f_{m l}
$$


Calculating the coefficients of the series (5.47) allows us to evaluate the integrals containing the product of $P n_{l}(x)$ and $\widetilde{P} n_{m}(x)$ :

$$
\begin{aligned}
& \int_{0}^{2 \mathbf{K}} \frac{\operatorname{sn}(x / 2)}{\operatorname{cd}(x / 2)} \tilde{P} n_{l}(x) P n_{m}(x) d x \\
& =\frac{(-1)^{m+l}}{k^{\prime}} \int_{0}^{2 \mathbf{K}} \frac{\mathrm{cn}(x / 2)}{\operatorname{sd}(x / 2)} \widetilde{P} n_{m}(x) P n_{l}(x) d x \\
& =\frac{(-1)^{l} 2 \mathbf{K}^{2} f_{m l}}{\sqrt{k^{\prime}} m \tanh (\lambda m)} .
\end{aligned}
$$

Taking into account Theorems 5.3 and 5.5, we deduce the fast convergent series

$$
\begin{aligned}
l \tanh (\lambda l) \frac{\mathrm{sn}^{2}(x / 2)}{\mathrm{cn}^{2}(x / 2)} P n_{l}(x) & =\sum_{m=1}^{\infty} \frac{(-1)^{m}}{\sqrt{k^{\prime}}} \widetilde{P} n_{m}(x) m \tanh (\lambda m) f_{l m}, \\
l \tanh (\lambda l) \frac{(-1)^{l}}{\sqrt{k^{\prime}}} \widetilde{P} n_{l}(x) & =\sum_{m=1}^{\infty} \frac{\mathrm{sn}^{2}(x / 2)}{\mathrm{cn}^{2}(x / 2)} P n_{m}(x) m \tanh (\lambda m) f_{l m} .
\end{aligned}
$$

One might use these findings to establish relations between coefficients of $\widetilde{P} n_{m}(x)$ - and $P n_{m}(x)$-series of the same function $f(x)$ in the form of fast convergent series. In this connection, the numbers $f_{m l}$ are especially important. These numbers have some interesting properties.

THEOREM 5.7. The numbers $f_{m l}$ have the bilinear expansion

$$
f_{m l}=\frac{(-1)^{m} \pi}{\mathbf{K} \sqrt{k^{\prime}}}+\frac{\pi^{2}(-1)^{l} l \sqrt{k^{\prime}} \operatorname{coth}(\lambda l)}{\mathbf{K}^{2} \cosh (\lambda m)} \sum_{n=1}^{\infty} \frac{\alpha_{m n} \beta_{l n}}{k^{2 n}} .
$$

The sets $f_{l m}$ and $f_{m l}$ are biorthonormal:

$$
\sum_{m=1}^{\infty} f_{r m} f_{m l}=\delta_{r l}
$$

If a sequence $u_{r}$ satisfies the condition $u_{r} \exp \left(-\lambda_{0} r\right) \in l_{1}, \lambda_{0}<2 \lambda, r=1,2, \ldots$, it may be represented in the form of the absolutely convergent series

$$
u_{r}=\sum_{m=1}^{\infty} \tilde{u}_{m} f_{r m}, \quad r=1,2, \ldots
$$

where the coefficients $\tilde{u}_{m}$ are defined as

$$
\tilde{u}_{m}=\sum_{l=1}^{\infty} u_{l} f_{m l}
$$


Proof. To establish (5.53), expand all functions in formula (5.46) into series in ascending powers of $\operatorname{sn}(x / 2)$ and equate coefficients of like powers. Upon invoking (2.1), (2.7), and (5.32), we find after some computations that

$$
\begin{gathered}
\frac{\pi}{\mathbf{K} k^{2 n}} \sum_{m=0}^{\infty} \frac{\alpha_{m n}}{\varepsilon_{m}}\left[(-1)^{m}+\frac{\mathbf{K} \sqrt{k^{\prime}} f_{m l}}{\pi}\right]=\frac{\pi(-1)^{l+1} l k^{\prime}}{\mathbf{K} k^{2 n}} \operatorname{coth}(\lambda l) \beta_{l n}, \quad n \geq 1, \\
\frac{\pi}{\mathbf{K}} \sum_{m=0}^{\infty} \frac{\alpha_{m 0}}{\varepsilon_{m}}\left[(-1)^{m}-\frac{\mathbf{K} \sqrt{k^{\prime}} f_{m l}}{\pi}\right]=0 .
\end{gathered}
$$

Then Theorem 3.5 gives the expansion (5.53).

In order to prove (5.54), multiply (5.51) by $\left(\operatorname{cn}(x / 2) / 2 \mathbf{K}^{2} \operatorname{sd}(x / 2)\right) P n_{m}(x)$ and integrate with respect to $x$ from 0 to $2 \mathbf{K}$. Applying the law of the mean to the integral representation (1.7) shows that $\left|(1 / x) \widetilde{P} n_{m}(x)\right| \leq D$, where $D$ does not depend on $x$ or $m$. On this ground, it is quite simple to conclude that the series is uniformly convergent with respect to $x$. Consequently, we are permitted to integrate termwise. Then the orthogonality property (5.2) and the integral (5.50) give the biorthonormal relation (5.54).

Validity of the representation (5.55) may be proved by inserting the expression (5.56). The absolute convergence of the resulting double series follows from the inequality $x^{2}+y^{2} \geq x^{\gamma} y^{2-\gamma}$ as $x \geq 1, y \geq 1,0 \leq \gamma \leq 2$, which implies that $f_{r m} f_{m l}=\exp (-\lambda \gamma m) \exp [-\lambda(2-\gamma) l] f$, where $f$ is a bounded quantity for every fixed $r$. Then interchanging the order of summation leads to the desired result which completes the proof.

Some more complex analysis shows that expanding into $f_{r m}$-series is possible for the faster increasing sequences.

THEOREM 5.8. Let $U(x) \in L_{1}[0,2 \mathrm{~K}]$ and

$$
\begin{aligned}
u_{r} & =\frac{\sqrt{k^{\prime}} \sinh ^{2}(\lambda r)}{2 \mathbf{K}^{2}} \int_{0}^{2 \mathbf{K}} U(x) \widetilde{P} n_{m}(x) d x \\
\tilde{u}_{m} & =\frac{(-1)^{m} \sinh ^{2}(\lambda m)}{2 \mathbf{K}^{2}} \int_{0}^{2 \mathbf{K}} U(x) P n_{m}(x) d x .
\end{aligned}
$$

Then the reciprocal expansions (5.55) and (5.56) are valid.

Proof. Multiply relations (5.47), (5.48), and (5.49) by $U(x)$ and integrate with respect to $x$ from 0 to $2 \mathbf{K}$. Formally, the theorem is obtained by integrating term by term and using the identity $(-1)^{m} \sinh ^{2}(\lambda m) f_{r m}=$ $(-1)^{r} \sinh ^{2}(r m) f_{m r}$. To justify this operation, we will show that the $n$th partial sums of the series in these relations are bounded uniformly with respect 
to $n$ and $x$. Since $\left|P n_{m}(x)\right| \leq 2 P n_{0}(x) \leq 2 \mathbf{K}$, we have

$$
\begin{aligned}
\frac{\mathbf{K} \sqrt{k^{\prime}}}{\pi}\left|\sum_{m=1}^{n} f_{m l} P n_{m}(x)\right| & =\left|\sum_{m=1}^{n}(-1)^{m}\left[\frac{\cosh 2 \lambda l}{\cosh 2 \lambda m+\cosh 2 \lambda l}-1\right] P n_{m}(x)\right| \\
& \leq 2 \mathbf{K} \sum_{m=1}^{\infty} \frac{\cosh 2 \lambda l}{\cosh 2 \lambda m+\cosh 2 \lambda l}+J
\end{aligned}
$$

where $J=\left|\sum_{m=1}^{n}(-1)^{m} P n_{m}(x)\right|$ may be estimated by substituting the integral representations for $P n_{m}(x)$. As $0 \leq x \leq \mathbf{K}$,

$$
\begin{aligned}
J & =\left|\int_{0}^{x} \frac{\mathrm{cn}(t / 2)}{\sqrt{\operatorname{sn}^{2}(x / 2)-\mathrm{sn}^{2}(t / 2)}}\left(\sum_{m=1}^{n}(-1)^{m} \cos \left(\mu_{m} t\right)\right) d t\right| \\
& =\left|\int_{0}^{x} \frac{\operatorname{cn}(t / 2)}{\sqrt{\operatorname{sn}^{2}(x / 2)-\operatorname{sn}^{2}(t / 2)}}\left(\frac{(-1)^{n} \cos ((2 n+1) \pi t / 4 \mathbf{K})}{2 \cos (\pi t / 4 \mathbf{K})}-\frac{1}{2}\right) d t\right| \\
& \leq \mathbf{K}+\int_{0}^{x} \frac{\operatorname{cn}(t / 2) d t}{2 \cos (\pi t / 4 \mathbf{K}) \sqrt{\operatorname{sn}^{2}(x / 2)-\operatorname{sn}^{2}(t / 2)}}
\end{aligned}
$$

The last integral is a continuous function in the indicated interval and therefore $J$ is bounded. As $\mathbf{K} \leq x \leq 2 \mathbf{K}$,

$$
\begin{aligned}
J & =\tanh (\lambda m)\left|\int_{x}^{2 \mathbf{K}} \frac{\mathrm{cn}(t / 2)}{\sqrt{\mathrm{sn}^{2}(x / 2)-\mathrm{sn}^{2}(t / 2)}}\left(\sum_{m=1}^{n}(-1)^{m} \sin \left(\mu_{m} t\right)\right) d t\right| \\
& \leq\left|\int_{x}^{2 \mathbf{K}} \frac{\operatorname{cn}(t / 2)}{\sqrt{\mathrm{sn}^{2}(x / 2)-\mathrm{sn}^{2}(t / 2)}} \frac{\sin ((n+1) \pi t / 4 \mathbf{K}) \cos (n \pi t / 4 \mathbf{K})}{2 \cos (\pi t / 4 \mathbf{K})} d t\right| \\
& \leq \int_{x}^{2 \mathbf{K}} \frac{\operatorname{cn}(t / 2) d t}{2 \cos (\pi t / 4 \mathbf{K}) \sqrt{\operatorname{sn}^{2}(x / 2)-\mathrm{sn}^{2}(t / 2)}}
\end{aligned}
$$

where the last integral again is a continuous function. Thus the considered partial sum is bounded. The same conclusion for the series containing $\widetilde{P} n_{m}(x)$ may be derived by a change $x=2 \mathbf{K}-x_{1}$. Then Lebesgue's theorem allows us to integrate term by term.

COROLlARY 5.9. If $r \exp (-2 \lambda r) u_{r} \in l_{1}$, then the expansions (5.55) and (5.56) are valid.

This result is obvious because one can take

$$
U(x)=\frac{\operatorname{sn}(x / 2)}{\operatorname{cd}(x / 2)} \sum_{m=1}^{\infty} \frac{(-1)^{m} u_{m}}{\sinh ^{2}(\lambda m)} m \tanh (\lambda m) P n_{m}(x) .
$$


6. Integral equations. The series expansions connecting $P n_{l}(x)$ and $\widetilde{P} n_{m}(x)$ have been deduced in Section 5. Of concern here are integral operators playing an analogous role. In particular, we will show that $P n_{l}(x)$ and $\widetilde{P} n_{m}(x)$ are eigenfunctions of certain Fredholm integral operators.

We multiply (5.33) by $\mathrm{cn}(t / 2)\left(\mathrm{cn}^{2}(t / 2)-\mathrm{cn}^{2}(s / 2)\right)^{-1 / 2}$ and integrate with respect to $t$ from 0 to $s, s<2 \mathbf{K}$. According to the integral representation (1.1) and the definition of the complete elliptic integral, we find the sum of the series

$$
S(x, s)=\sum_{m=1}^{\infty} \frac{P n_{m}(x) P n_{m}(s)}{2 \cosh (\lambda m)}
$$

namely,

$$
S(x, s)=\mathbf{K}(k) \mathbf{K}\left(k \operatorname{sn} \frac{x}{2} \operatorname{sn} \frac{s}{2}\right)-\mathbf{K}\left(k \operatorname{sn} \frac{x}{2}\right) \mathbf{K}\left(k \operatorname{sn} \frac{s}{2}\right) .
$$

Then recourse to the proven theorems yields the Fredholm integral equation for $P n_{m}(x)$ which can be written in the symmetric form

$$
\lambda_{m} \mathfrak{O} \mathfrak{n}_{m}(s)=\frac{1}{\mathbf{K}^{2}} \int_{0}^{2 \mathbf{K}} \sqrt{\operatorname{sn}(x / 2) \operatorname{sn}(s / 2) / \operatorname{cd}(x / 2) \operatorname{cd}(s / 2)} S(x, s) \not \mathfrak{O n} \mathfrak{n}_{m}(x) d x,
$$

where the orthonormal functions $\not \mathfrak{O n} \mathfrak{n}_{m}(s)$ are defined before and $\lambda_{m}^{-1}=$ $m \sinh \lambda m$. The spectrum of this operator is simple because its eigenfunctions form an orthonormal basis in $L_{2}[0,2 \mathrm{~K}]$.

Prove that the kernel of the equation is positive for $0 \leq x, s<2 \mathbf{K}$. It is seen that this statement is equivalent to the monotonic decrease of the function $\mathbf{K}(a t) / \mathbf{K}(t), 0<a<1$, for $0<t<1$. If $(d / d t) t(d / d t)(\ln f(t))>0$ on some interval, then any positive function of the form $f(a t) / f(t)$ decreases monotonically. Show that the complete elliptic integral $\mathbf{K}(t)$ meets this condition for $0<t<1$. Indeed, by using the differential equation for $\mathbf{K}(t)$ [5], we obtain after simple manipulations

$$
\frac{d}{d t}\left(\frac{t(d / d t) \mathbf{K}(t)}{\mathbf{K}(t)}\right)=t \frac{(\mathbf{K}(t)+t(d / d t) \mathbf{K}(t))^{2}-((d / d t) \mathbf{K}(t))^{2}}{\left(1-t^{2}\right) \mathbf{K}^{2}(t)}
$$

Now the inequalities $d \mathbf{K}(t) / d t>0$ and

$$
\mathbf{K}(t)-(1-t) \frac{d \mathbf{K}(t)}{d t}=\int_{0}^{1} \frac{1-t x^{2}}{\sqrt{1-x^{2}}\left(1-t^{2} x^{2}\right)^{3 / 2}} d x>0, \quad|t|<1
$$

enable us to conclude that the function (6.4) is positive for $0<t<1$.

An integral operator transforming $P n_{m}(x)$ into $\widetilde{P} n_{m}(x)$ and $\left(\widetilde{P} n_{m}(x)\right.$ into $P n_{m}(x)$ ) can be derived from (6.3) by setting $x=2 \mathbf{K}-x$ and $s=2 \mathbf{K}-s$. Analogous Volterra integral operators will be pointed out below. 
Going back to (1.7), we write the Fourier series

$$
\sum_{m=1}^{\infty} \tanh (\lambda m) P n_{m}(x) \sin \left(\mu_{m} t\right)= \begin{cases}0, & t<x, \\ \frac{\mathbf{K c n}(t / 2)}{\sqrt{\operatorname{sn}^{2}(t / 2)-\mathrm{sn}^{2}(x / 2)}}, & t>x .\end{cases}
$$

Upon multiplying by $\mathrm{sn}(t / 2)\left(\mathrm{cn}^{2}(t / 2)-\mathrm{cn}^{2}(s / 2)\right)^{-1 / 2}$ and integrating with respect to $t$ from 0 to $s$, we find

$$
\sum_{m=1}^{\infty} \tanh ^{2}(\lambda m) P n_{m}(x) \widetilde{P} n_{m}(s)= \begin{cases}-\frac{2 \mathbf{K}}{\operatorname{dn}(x / 2)} \mathbf{K}\left(\sqrt{1-\frac{\mathrm{dn}^{2}(s / 2)}{\mathrm{dn}^{2}(x / 2)}}\right), & s>x, \\ 0, & s<x\end{cases}
$$

and after that

$$
\widetilde{P} n_{m}(s)=-\frac{m}{\mathbf{K} \tanh (\lambda m)} \int_{0}^{s} P n_{m}(x) \frac{\operatorname{sn}(x / 2)}{\operatorname{cn}(x / 2)} \mathbf{K}\left(\sqrt{1-\frac{\mathrm{dn}^{2}(s / 2)}{\operatorname{dn}^{2}(x / 2)}}\right) d x
$$

The inverse operator is determined in the same way from the Fourier series (5.32) which leads to

$$
\begin{aligned}
\sum_{m=1}^{\infty} P n_{m}(x) \widetilde{P} n_{m}(s)= & -\frac{2}{\operatorname{dn}(s / 2)} \mathbf{K}\left(k \operatorname{sn} \frac{x}{2}\right) \mathbf{K}\left(k \operatorname{cd} \frac{s}{2}\right) \\
& + \begin{cases}\frac{2 \mathbf{K}}{\operatorname{dn}(s / 2)} \mathbf{K}\left(\sqrt{\left.1-\frac{\mathrm{dn}^{2}(x / 2)}{\operatorname{dn}^{2}(s / 2)}\right),}\right. & s<x, \\
0, & s>x,\end{cases}
\end{aligned}
$$

and after that to the relation

$$
\begin{aligned}
P n_{m}(x)= & \frac{m \tanh (\lambda m)}{\mathbf{K}} \int_{0}^{x} \widetilde{P} n_{m}(s) \frac{\operatorname{cn}(s / 2)}{\operatorname{sn}(s / 2)} \mathbf{K}\left(\sqrt{1-\frac{\mathrm{dn}^{2}(x / 2)}{\operatorname{dn}^{2}(s / 2)}}\right) d s \\
& +C \mathbf{K}\left(k \operatorname{sn} \frac{x}{2}\right) .
\end{aligned}
$$

The constant number $C$ can be found by proceeding to the limit as $x \rightarrow 0$. We observe from (1.6) and (1.8) that $P n_{m}(x) \sim \pi+O\left(x^{2}\right)$ and $\widetilde{P} n_{m}(x) \sim O\left(x^{2}\right)$. Then

$$
C=-\frac{m \tanh (\lambda m)}{\mathbf{K}^{2}} \int_{0}^{2 \mathbf{K}} \widetilde{P} n_{m}(s) \frac{\operatorname{cn}(s / 2)}{\operatorname{sn}(s / 2)} \mathbf{K}\left(k \mathrm{~cd} \frac{s}{2}\right) d s=2
$$


It is readily seen that another pair of the Volterra operators is derived from the series (6.7) and (6.9):

$$
\begin{aligned}
P n_{m}(x)= & -\frac{m}{\mathbf{K} \tanh (\lambda m)} \int_{x}^{2 \mathbf{K}} \widetilde{P} n_{m}(s) \frac{\operatorname{cn}(s / 2)}{\operatorname{sd}(s / 2) \operatorname{dn}(x / 2)} \mathbf{K}\left(\sqrt{1-\frac{\mathrm{dn}^{2}(s / 2)}{\operatorname{dn}^{2}(x / 2)}}\right) d s, \\
\tilde{P} n_{m}(s)= & \frac{m \tanh (\lambda m)}{\mathbf{K}} \int_{s}^{2 \mathbf{K}} P n_{m}(x) \frac{\operatorname{sn}(x / 2)}{\operatorname{cd}(x / 2) \operatorname{dn}(s / 2)} \mathbf{K}\left(\sqrt{1-\frac{\operatorname{dn}^{2}(x / 2)}{\operatorname{dn}^{2}(s / 2)}}\right) d x \\
& +2 \frac{(-1)^{m}}{\operatorname{dn}(s / 2)} \mathbf{K}\left(k \operatorname{cd} \frac{s}{2}\right) .
\end{aligned}
$$

Combining (6.8) and (6.13) coupled with changing variables yields, for $\mathfrak{b} \mathfrak{n}_{m}(x)$, another Fredholm integral equation with the symmetric continuous kernel

$$
\begin{aligned}
& \mathfrak{P n _ { m }}(s)=(-1)^{m} \frac{m \sinh (2 \lambda m)}{2 \mathbf{K}^{2}} \int_{0}^{2 \mathbf{K}} \sqrt{\frac{\operatorname{sn}(x / 2) \operatorname{sn}(s / 2)}{\operatorname{cd}(x / 2) \operatorname{cd}(s / 2)}} S_{1}(x, s) \mathfrak{\bullet \mathfrak { n } _ { m }}(x) d x, \\
& S_{1}(x, s)=\mathbf{K}(k) \mathbf{K}\left(\sqrt{\left|1-\frac{\mathrm{dn}^{2}(x / 2) \mathrm{dn}^{2}(s / 2)}{1-k^{2}}\right|}\right)-\mathbf{K}\left(k \operatorname{sn} \frac{x}{2}\right) \mathbf{K}\left(k \operatorname{sn} \frac{s}{2}\right) .
\end{aligned}
$$

Our transformation of $S_{1}(x, s)$ to this form rests on the well-known relations for the complete elliptic integral [5].

7. Differential equations and zeros. We denote

$$
Y_{m}(u)=k \operatorname{sn} \frac{x}{2} P n_{m}(x), \quad u=\left(k \operatorname{sn} \frac{x}{2}\right)^{-2}
$$

The functions $Y_{m}(u)$ form an orthogonal basis in $L_{2}\left[k^{-2}, \infty\right]$. According to (1.6), their integral representation can be written in the form

$$
Y_{m}(u)=2 \cosh (\lambda m) \int_{0}^{\mathrm{K}} \frac{\mathrm{dn} s}{\sqrt{u-\mathrm{sn}^{2} s}} \cos \frac{\pi m s}{\mathbf{K}} d s .
$$

Integration by parts shows that $Y_{m}(u)$ is a solution of the Heun differential equation [3, 9]

$$
\frac{d^{2} Y}{d u^{2}}+\left(\frac{\gamma}{u}+\frac{\delta}{u-1}+\frac{\varepsilon}{u-a}\right) \frac{d Y}{d u}+\frac{\alpha \beta u-q}{u(u-1)(u-a)} Y=0
$$


where $\gamma=\delta=1, \varepsilon=0, \alpha=\beta=1 / 2, q=(1 / 4)\left(1-4 \mu_{m}^{2}\right) k^{-2}$, and $a=k^{-2}$. If we assume that $Y(a, q, \alpha, \beta, \gamma, \delta, u), \varepsilon=\alpha+\beta-\gamma-\delta+1$, is a solution of the form $Y(u)=1+\sum_{n=1}^{\infty} a_{n} u^{n}$, then the function $Y(u)=A(a / u)^{\alpha} Y\left(a, q_{1}, \alpha, \alpha-\right.$ $\gamma+1, \alpha-\beta+1, \varepsilon, a / u), q_{1}=q+\alpha[(\varepsilon-\beta) a+\delta-\beta]$, is a solution of Heun's differential equation (7.3) as well. Identifying the series (2.1), one can find that

$$
\begin{aligned}
& P n_{m}(x)=\pi Y\left(\frac{1}{k^{2}}, \frac{1}{4}-\frac{\mu_{m}^{2}}{k^{2}}, \frac{1}{2}, \frac{1}{2}, 1,0, \operatorname{sn}^{2} \frac{x}{2}\right), \\
& \widetilde{P} n_{m}(x)=\frac{(-1)^{m+1} \pi}{\operatorname{dn}(x / 2)} Y\left(\frac{1}{k^{2}}, \frac{1}{4}-\frac{\mu_{m}^{2}}{k^{2}}, \frac{1}{2}, \frac{1}{2}, 1,0, \mathrm{~cd}^{2} \frac{x}{2}\right) .
\end{aligned}
$$

Another form of expression in terms of the solutions of Heun's equation follows from the integral representation (1.8) and the series (2.7):

$$
\begin{aligned}
& P n_{m}(x)=\frac{(-1)^{m+1} \pi^{2} m k^{\prime}}{\mathbf{K} \tanh (\lambda m) \operatorname{dn}(x / 2)} \operatorname{cd}^{2} \frac{x}{2} Y\left(\frac{1}{k^{2}}, 1+\frac{1-\mu_{m}^{2}}{k^{2}}, \frac{3}{2}, \frac{3}{2}, 2,1, \operatorname{cd}^{2} \frac{x}{2}\right), \\
& \widetilde{P} n_{m}(x)=-\frac{\pi^{2} m}{\mathbf{K} \tanh (\lambda m)} \operatorname{sn}^{2} \frac{x}{2} Y\left(\frac{1}{k^{2}}, 1+\frac{1-\mu_{m}^{2}}{k^{2}}, \frac{3}{2}, \frac{3}{2}, 2,1, \operatorname{sn}^{2} \frac{x}{2}\right) .
\end{aligned}
$$

Thus $P n_{m}(x)$ and $\widetilde{P} n_{m}(x), m=1,2, \ldots$, are complete sets of the eigenfunctions of the singular Sturm-Liouville problems for Heun's equation (the Heun functions [3, 9]). Therefore, $P n_{m}(x)$ has $m$ zeros $x_{n}<x_{n+1}, x_{m}=2 \mathbf{K}$. All $m-1$ zeros, that lie within $(0,2 \mathbf{K})$, are simple. The zeros of the contiguous functions $P n_{m}(x)$ and $P n_{m+1}(x)$ alternate. It is seen from (2.1) and (2.7) that $P n_{m}(x)$ is positive at point $x=0$ and $(-1)^{m+1} P n_{m}(x)$ is positive in the vicinity of $x=2 \mathbf{K}$. Then a simple analysis of (6.8) brings in the conclusion that the smallest zero of $P n_{m}(x)$ is less than the zeros of $\widetilde{P} n_{m}(x)$. Consequently, all zeros $x_{n}, n<m$, are localized in the interval $\left[x_{1}, 2 \mathbf{K}-x_{1}\right)$ and $x_{1}<\mathbf{K}$. We also note that the theory of Heun's equation is an additional source of relations for the functions treated here [9].

8. On Legendre functions of complex degree. In the simultaneous limits $k \rightarrow 1$ and $\mu_{m} \rightarrow \xi$, the function $P n_{m}(x)$ becomes the Legendre function

$$
\pi \cosh \frac{x}{2} P_{-1 / 2+i \xi}(\cosh x)
$$

The series (2.1) turns into the series

$$
P_{-1 / 2+i \xi}(\cosh x)=\frac{1}{\cosh (x / 2)} \sum_{n=0}^{\infty}(-1)^{n} \alpha_{n}(\xi) \frac{(2 n-1) ! !}{(2 n) ! !} \tanh ^{2 n} \frac{x}{2}
$$


whose coefficients have the generated function

$$
\cosh \frac{x}{2} \operatorname{Re}[\exp (i \xi)]=\sum_{n=0}^{\infty} \alpha_{n}(\xi)\left(i \tanh \frac{x}{2}\right)^{2 n}
$$

Then these coefficients are the even Pollaczek polynomials [3]

$$
\alpha_{n}(\xi)=P_{2 n}^{1 / 2}\left(\xi, \frac{\pi}{2}\right)=(-1)^{n} F\left(-2 n, \frac{1}{2}+i \xi, 1,2\right)
$$

which are orthogonal for $\xi \in[0, \infty)$ :

$$
2 \int_{0}^{\infty} \frac{\alpha_{n}(\xi) \alpha_{r}(\xi)}{\cosh (\pi \xi)} d \xi=\delta_{n r}
$$

Their Fourier transform representation can be derived from (2.2) by taking limits $k \rightarrow 1$ and $\mu_{m} \rightarrow \xi$ :

$$
\begin{aligned}
\alpha_{n}(\xi) & =(-1)^{n} \frac{\cosh (\pi \xi)}{\pi} \int_{0}^{\infty} \frac{\tanh ^{2 n}(t / 2)}{\operatorname{cn}(t / 2)} \cos (\xi t) d t \\
& =\operatorname{Re} \frac{2(2 n) !(-1)^{n}}{\Gamma(2 n+3 / 2-i \xi) \Gamma(1 / 2+i \xi)} F\left(\frac{1}{2}-i \xi, 2 n+1,2 n+\frac{3}{2}-i \xi,-1\right)
\end{aligned}
$$

The expansion (5.19) becomes the Mehler-Fok integral transform [7]

$$
\begin{aligned}
& f(s)=\int_{0}^{\infty} F(\xi) \xi P_{-1 / 2+i \xi}(\cosh s) \tanh (\pi \xi) d \xi \\
& F(\xi)=\int_{0}^{\infty} f(x) P_{-1 / 2+i \xi}(\cosh x) \sinh x d x
\end{aligned}
$$

One important Mehler-Fok integral may be evaluated by employing the series (8.2):

$$
\int_{0}^{\infty} \frac{\alpha_{n}(\xi)}{\cosh \pi \xi} P_{-1 / 2+i \xi}(\cosh x) d \xi=(-1)^{n} \frac{(2 n-1) ! !}{2(2 n) ! ! \cosh (x / 2)} \tanh ^{2 n} \frac{x}{2}
$$

In the same limits, the function $\widetilde{P} n_{m}(x)$ converts into

$$
-\pi \cosh \frac{x}{2} \operatorname{coth}(\pi \xi) P^{-}(\xi, x),
$$

where $P^{-}(\xi, x)$ is the imaginary part of the Legendre function $P_{i \xi}(\cosh x)$. The function $P^{-}(\xi, x)$, as it indicates (2.7), may be expanded into the series in 
ascending powers of $\tanh ^{2}(x / 2)$ with coefficients expressed in terms of the Pollaczek polynomials $P_{2 n}^{1}(\xi, \pi / 2)$. One might see from the preceding discussion that $P^{-}(\xi, x)$ and $P_{1 / 2+i \xi}(\cosh x)$ are related by the transforming operators

$P^{-}(\xi, x)$

$$
\begin{aligned}
& \quad=\frac{\xi}{\pi \cosh (x / 2)} \int_{0}^{x} P_{-1 / 2+i \xi}(\cosh t) \sinh t \mathbf{K}\left(\frac{\sqrt{\cosh ^{2}(x / 2)-\cosh ^{2}(t / 2)}}{\cosh (x / 2)}\right) d t, \\
& P_{-(1 / 2)+i \xi(\cosh t)} \\
& \quad=\frac{2 \xi}{\pi \tanh ^{2}(\pi \xi)} \int_{x}^{\infty} \frac{P^{-}(\xi, x)}{\sinh (t / 2)} \mathbf{K}\left(\frac{\sqrt{\cosh ^{2}(t / 2)-\cosh ^{2}(x / 2)}}{\cosh (t / 2)}\right) d t .
\end{aligned}
$$

The formula (5.49) hints at the existence of its continual analog

$$
P_{-1 / 2+i \xi}(\cosh x)=2 \int_{0}^{\infty} \frac{\sinh 2 \pi \eta}{\cosh 2 \pi \eta+\cosh 2 \pi \xi} P^{-}(\eta, x) d \eta
$$

This, indeed, can be confirmed by contour integration.

Upon proceeding to the limit in the expansion (5.31), we also deduce the new integral transform, namely,

$$
\begin{aligned}
g(s) & =\int_{0}^{\infty} G^{-}(\xi) P^{-}(\eta, s) \xi \operatorname{coth}(\pi \xi) d \xi, \\
G^{-}(\xi) & =2 \int_{0}^{\infty} g(x) P^{-}(\eta, x) \operatorname{coth} \frac{x}{2} d x .
\end{aligned}
$$

This result requires the more rigorous treatment fulfilled in [8].

Employing (8.12) to (8.11), we obtain an integral containing $P_{-1 / 2+i \xi}(\cosh x)$. This integral may be interpreted as the Mehler-Fok transform. Its inversion gives us the following continual analog of the series representation (5.52):

$$
P^{-}(\eta, x)=4 \frac{\sinh ^{2}(\pi \eta) \sinh ^{2}(x / 2)}{\eta} \int_{0}^{\infty} \frac{\tanh (\pi \xi) P_{-1 / 2+i \xi}(\cosh x)}{\cosh 2 \pi \eta+\cosh 2 \pi \xi} \xi d \xi
$$

9. Dual series equations involving trigonometric functions. Many of periodic and doubly periodic mixed boundary value problems faced by engineers, physicists, and applied mathematicians are reduced to dual series equations 
of the form

$$
\begin{gathered}
\sum_{m=0}^{\infty} \frac{\tanh (\lambda m)}{m \varepsilon_{m}}[1+L(\lambda m)] A_{m} \cos \left(\mu_{m} t\right)=f(t), \quad 0 \leq t \leq a, \\
\sum_{m=0}^{\infty} \frac{1}{\varepsilon_{m}} A_{m} \cos \left(\mu_{m} t\right)=0, \quad a \leq t \leq 2 \mathbf{K}, \\
\sum_{m=1}^{\infty} \frac{\tanh (\lambda m)}{m}[1+L(\lambda m)] B_{m} \sin \left(\mu_{m} t\right)=g(t), \quad 0 \leq t \leq a, \\
\sum_{m=1}^{\infty} B_{m} \sin \left(\mu_{m} t\right)=0, \quad a \leq t \leq 2 \mathbf{K},
\end{gathered}
$$

where $A_{m}$ and $B_{m}$ are unknown coefficients satisfying the conditions

$$
F(t)=\sum_{m=0}^{\infty} \frac{1}{\varepsilon_{m}} A_{m} \cos \left(\mu_{m} t\right) \in L_{1}, \quad G(t)=\sum_{m=1}^{\infty} B_{m} \sin \left(\mu_{m} t\right) \in L_{1} ;
$$

$f(t)$ and $g(t)$ are given continuous and piecewise continuously differentiable functions; $1+L(s)$ is a given nonnegative bounded function, $L(\lambda m)=\eta / m+$ $O\left(1 / m^{2}\right)$ as $m \rightarrow \infty$.

A number of methods reducing trigonometric dual series equations to equations of the second kind with completely continuous operators is well known. Arising in the examined case here, operator equations have a general shortcoming-their $L_{2}$-norms tend to zero as $\lambda \rightarrow 0$. Thus these equations are illconditioned for small $\lambda$ and may be unsuitable for mathematical interpretation or numerical computation. We suggest here the method of reducing the considered dual series equations to the equivalent Fredholm integral equation of the second kind which is well-conditioned if $1+L(\lambda m)$ is not small. Our approach is based on employing the integral representations for the function $P n_{m}(s)$ and the theory of the orthogonal expansions developed above.

To solve (9.1) and (9.2), we note that

$$
\begin{aligned}
& A_{m}=\frac{1}{\mathbf{K}} \int_{0}^{a} F(t) \cos \left(\mu_{m} t\right) d t=A_{0}+\mu_{m} \widetilde{A}_{m}, \\
& \widetilde{A}_{m}=-\frac{1}{\mathbf{K}} \int_{0}^{a}\left[\int_{t}^{a} F(u) d u\right] \sin \left(\mu_{m} t\right) d t .
\end{aligned}
$$

Then

$$
\begin{gathered}
\frac{\pi}{2 \mathbf{K}} \sum_{m=1}^{\infty} \widetilde{A}_{m} \tanh (\lambda m)[1+L(\lambda m)] \cos \left(\mu_{m} t\right)=f_{1}(t), \quad 0 \leq t \leq a, \\
\sum_{m=1}^{\infty} \widetilde{A}_{m} \sin \left(\mu_{m} t\right)=0, \quad a \leq t \leq 2 \mathbf{K},
\end{gathered}
$$


where

$$
f_{1}(t)=f(t)-A_{0} \sum_{m=0}^{\infty} \frac{\tanh (\lambda m)}{m \varepsilon_{m}}[1+L(\lambda m)] \cos \left(\mu_{m} t\right) .
$$

We introduce the operators

$$
\begin{aligned}
& \mathbf{R}_{1}(\cdot)=\frac{1}{\pi \mathbf{K}} \int_{0}^{x}(\cdot) \frac{\mathrm{cn}(t / 2)}{\sqrt{\mathrm{cn}^{2}(t / 2)-\mathrm{cn}^{2}(x / 2)}} d t \\
& \mathbf{R}_{2}(\cdot)=\frac{1}{2 \mathbf{K}^{2}} \int_{x}^{2 \mathbf{K}}(\cdot) \frac{\mathrm{cn}(t / 2)}{\sqrt{\mathrm{cn}^{2}(t / 2)-\mathrm{cn}^{2}(x / 2)}} d t .
\end{aligned}
$$

In accordance with the representation (1.1) and (1.7), these operators transform trigonometric functions into $P n_{m}(x)$. If (9.7) is subjected to the action of the operator $\mathbf{R}_{1}$ and (9.8) is subjected to the action of the operator $\mathbf{R}_{1}$, then these equations take the form

$$
\begin{gathered}
\frac{1}{2 \mathbf{K}^{2}} \sum_{m=1}^{\infty} \tilde{A}_{m} \tanh (\lambda m)[1+L(\lambda m)] P n_{m}(x)=\mathbf{R}_{1}\left(f_{1}\right), \quad 0 \leq x \leq a, \\
\frac{1}{2 \mathbf{K}^{2}} \sum_{m=1}^{\infty} \tilde{A}_{m} \tanh (\lambda m) P n_{m}(x)=0, \quad a \leq t \leq 2 \mathbf{K} .
\end{gathered}
$$

We will seek a solution of the dual series equations of the form

$$
\tilde{A}_{m}=m \int_{0}^{a} \sqrt{\frac{\operatorname{sn}(s / 2)}{\operatorname{cd}(s / 2)}} \omega(s) P n_{m}(s) d s, \quad m=1,2, \ldots,
$$

where $\omega(s)$ is an unknown absolutely integrable function. Substitute (9.13) into (9.12). According to the inversion theorem for $P n_{m}$-series, this equation is satisfied identically. The relations (9.11), (9.13), and the inversion theorem lead to the Fredholm integral equation of the second kind

$$
\omega(x)+\int_{0}^{a} \omega(s) H(x, s) d s=\sqrt{\frac{\operatorname{sn}(x / 2)}{\operatorname{cd}(x / 2)}}\left[\mathbf{R}_{1}(f)-A_{0} H(x)\right], \quad 0 \leq x \leq a,
$$

where the symmetric kernel $H(x, s)$ and the function $H(x)$ are given by

$$
\begin{gathered}
H(x, s)=\sum_{m=1}^{\infty} L(\lambda m) \mathcal{P} \mathfrak{n}_{m}(x) \mathcal{P} \mathfrak{n}_{m}(s), \\
H(x)=\frac{\lambda \mathbf{K}(k \operatorname{sn}(x / 2))}{\pi \mathbf{K}}+\sum_{m=1}^{\infty} \frac{\tanh (\lambda m)}{\pi m \mathbf{K}}[1+L(\lambda m)] P n_{m}(x) .
\end{gathered}
$$

It follows from the asymptotic expansions of $P n_{m}(x)$ and $L(\lambda m)$ that the kernel $H(x, s)$ has a logarithmic singularity on the line $x=s$ when $\eta \neq 0$. As $\eta=0$, the kernel is a continuous function. Let $\mathbf{H}$ be the integral operator in 
(9.14) and let I be the unit operator. Using the Parseval formula (5.5), we obtain the estimates

$$
\begin{aligned}
((\mathbf{I}+\mathbf{H}) \omega, \omega) & =\sum_{m=1}^{\infty}[1+L(\lambda m)]\left(\omega, \not \mathfrak{O n} \mathfrak{n}_{m}\right)^{2} \\
& >\min [1+L(\lambda m)]\|\omega\|^{2} \geq 0, \\
\|\mathbf{I}+\mathbf{H}\| & =\sup \frac{((\mathbf{I}+\mathbf{H}) \omega, \omega)}{\|\omega\|^{2}}<\max [1+L(\lambda m)] .
\end{aligned}
$$

We see that the operator $\mathbf{I}+\mathbf{H}$ is positive. Hence the Fredholm integral equation has the unique solution. If the number $\min [1+L(\lambda m)]$ is not small and the number $\max [1+L(\lambda m)]$ is not large, then the equation is well conditioned and can be solved by numerical methods. Since the right-hand side of the equation (9.14) is an absolutely integrable function, the solution $\omega(x)$ also is absolutely integrable, as it was assumed. Moreover, $\omega(x) \sqrt{\operatorname{sn}(x / 2)}$ is differentiable on $(0, a]$ and its derivative has a logarithmic singularity at the point $x=0$.

Evaluating $F(t)$ yields

$$
\begin{aligned}
F(t) & =-\frac{2 \mathbf{K}}{\pi} \frac{d^{2}}{d t^{2}} \sum_{m=1}^{\infty} \frac{1}{m} \tilde{A}_{m} \cos \left(\mu_{m} t\right) \\
& =-\frac{2 \mathbf{K}}{\pi} \frac{d^{2}}{d t^{2}} \int_{t}^{a}\left[\frac{\mathbf{K c n}(t / 2)}{\sqrt{\operatorname{cn}^{2}(t / 2)-\mathrm{cn}^{2}(s / 2)}}-\mathbf{K}\left(k \operatorname{sn} \frac{s}{2}\right)\right] \sqrt{\frac{\operatorname{sn}(s / 2)}{\operatorname{cd}(s / 2)}} \omega(s) d s \\
& =3 \mathbf{K}^{2}\left(\frac{\operatorname{cn}(a / 2) \operatorname{sn}(a / 2) \operatorname{dn}(a / 2)}{\operatorname{cn}^{2}(t / 2)-\operatorname{cn}^{2}(a / 2)}\right)^{3 / 2} \omega(a)+F_{1}(t), \quad F_{1}(t) \in L_{1}
\end{aligned}
$$

Then the condition (9.15) gives a complementary relation which enables us to determine $A_{0}$

$$
\omega(a)=0
$$

Since functions from $L^{1}$ do not belong to the null-spaces of the operators $\mathbf{R}_{1}$ and $\mathbf{R}_{2}$, these operators are left equivalent transformations. The substitution (9.13) together with the complementary condition (9.19) may be interpreted as a right equivalent regularization. Thus we achieve the following theorem.

THEOREM 9.1. The dual integral equations (9.7) and (9.8) are equivalent to the Fredholm integral equation of the second kind (9.14) coupled with the condition (9.19).

When $L(\lambda m) \equiv 0$, the integral operators do not occur, and the numbers $A_{m}$ and $B_{m}$ are determined explicitly. 
The dual equations (9.3) and (9.4) can be treated by the same argument as (9.7) and (9.8). The only difference is that a solution scheme uses the operator

$$
\mathbf{R}(\cdot)=\frac{1}{\pi \mathbf{K}} \frac{d}{d x} \int_{0}^{x}(\cdot) \frac{\operatorname{sn}(t / 2) \mathrm{dn}(t / 2)}{\sqrt{\mathrm{cn}^{2}(t / 2)-\mathrm{cn}^{2}(x / 2)}} d t
$$

in place of the operator $\mathbf{R}_{1}$. Finally, the dual series equations are reduced to the Fredholm integral equation

$$
\omega(x)+\int_{0}^{a} \omega(s) H(x, s) d s=\sqrt{\frac{\operatorname{sn}(x / 2)}{\operatorname{cd}(x / 2)}} \mathbf{R}(g), \quad 0 \leq x \leq a,
$$

whose kernel is indicated above. As above, we have the following theorem.

THEOREM 9.2. The dual integral equations (9.3) and (9.4) are equivalent to the Fredholm integral equation of the second kind (9.21).

When $L(\lambda m) \equiv 0$, we again obtain the exact solution of the equation and the numbers $B_{m}$ are exactly determined as well.

\section{REFERENCES}

[1] G. Alexits, Convergence Problems of Orthogonal Series, International Series of Monographs in Pure and Applied Mathematics, vol. 20, Pergamon Press, New York, 1961, translated from the German by I. Földer.

[2] G. D. Birkhoff and G.-C. Rota, On the completeness of Sturm-Liouville expansions, Amer. Math. Monthly 67 (1960), 835-841.

[3] A. Erdélyi, W. Magnus, F. Oberhettinger, and F. G. Tricomi, Higher Transcendental Functions. Vols. I, II, McGraw-Hill, New York, 1953.

[4] P. Fatou, Séries trigonométriques et séries de Taylor, Acta Math. 30 (1906), 335400.

[5] I. S. Gradshteyn and I. M. Ryzhik, Table of Integrals, Series, and Products, Academic Press, New York, 1980.

[6] K. Iséki, On complete orthonormal sets in Hilbert space, Proc. Japan Acad. 33 (1957), 450-452.

[7] N. N. Lebedev, Special Functions and Their Applications, revised English ed., Prentice-Hall, New Jersey, 1965, translated and edited by Richard A. Silverman.

[8] P. Malits, On Legendre functions of imaginary degree and associated integral transforms, J. Math. Anal. Appl. 281 (2003), no. 1, 205-220.

[9] A. Ronveaux (ed.), Heun's Differential Equations, Oxford Science Publications, The Clarendon Press, Oxford University Press, New York, 1995.

[10] G. N. Watson, A Treatise on the Theory of Bessel Functions, Cambridge University Press, Cambridge, 1966.

[11] E. T. Whittaker and G. N. Watson, A Course of Modern Analysis, Cambridge University Press, Cambridge, 1965.

P. Malits: Department of Communication Engineering and Center for Applied and Industrial Mathematics at Department of Sciences, Holon Academic Institute of Technology, 52 Golomb Street, Holon 58102, Israel

E-mail address: ma1its@hait.ac. 11 


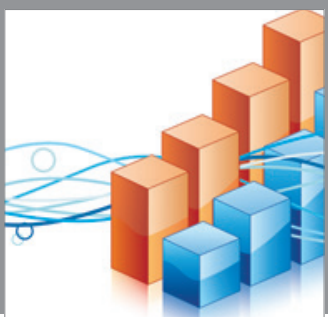

Advances in

Operations Research

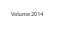

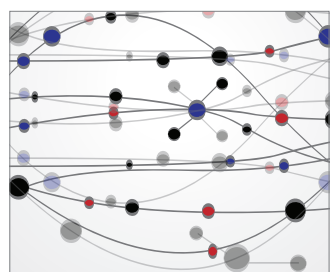

\section{The Scientific} World Journal
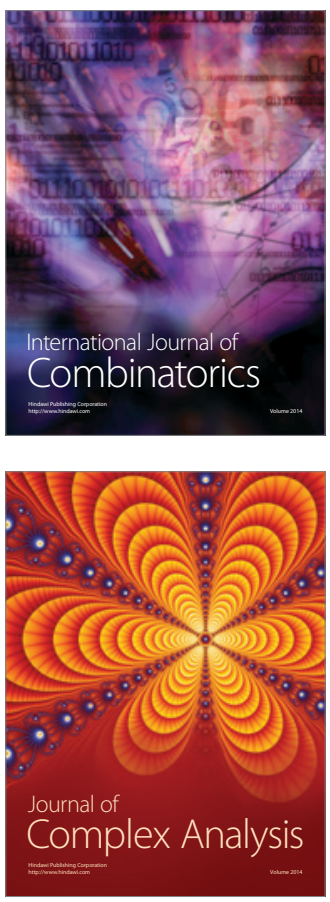

International Journal of

Mathematics and

Mathematical

Sciences
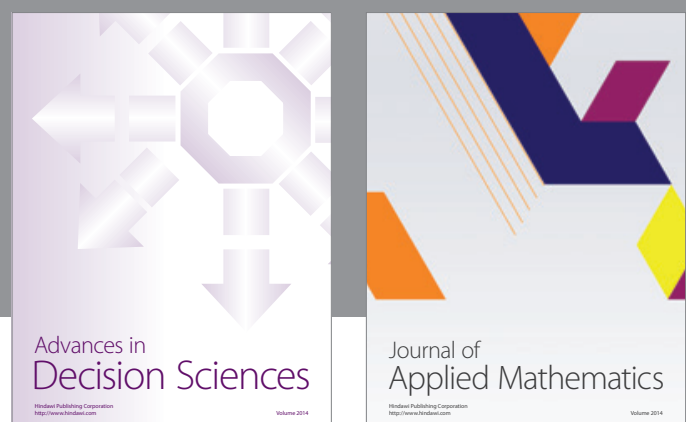

Journal of

Applied Mathematics
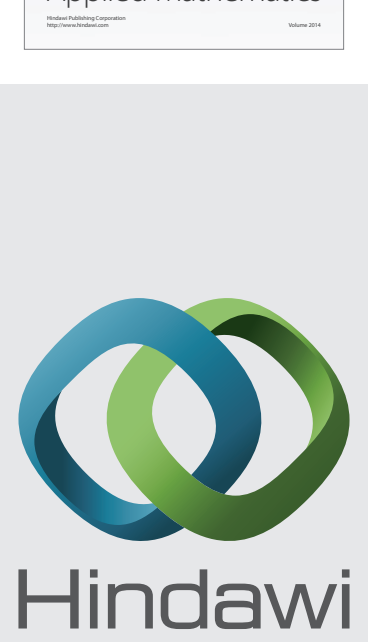

Submit your manuscripts at http://www.hindawi.com
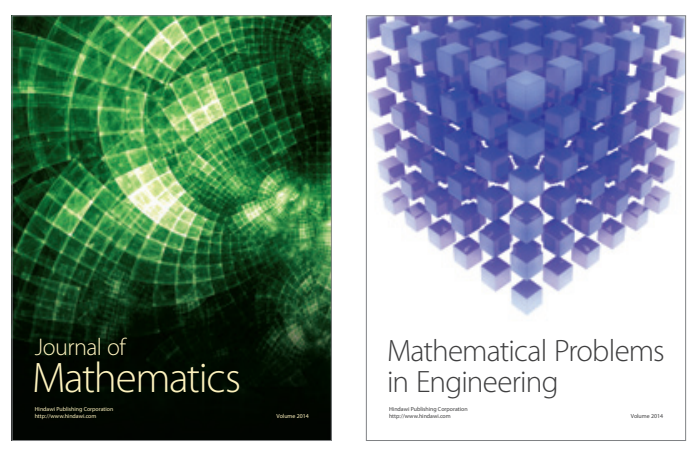

Mathematical Problems in Engineering
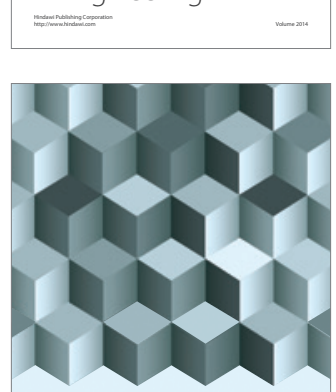

Journal of

Function Spaces
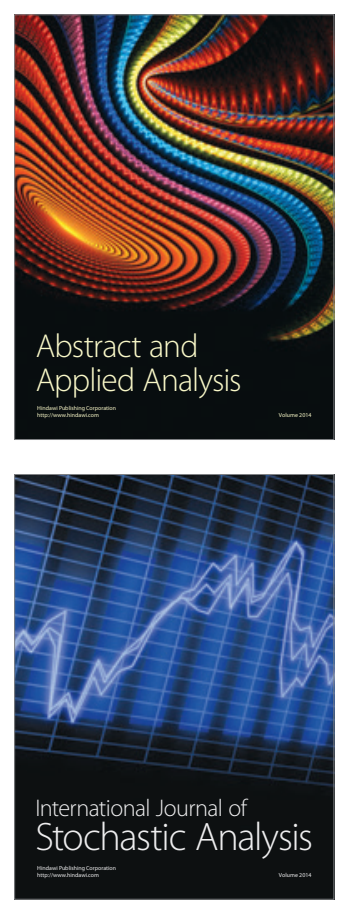

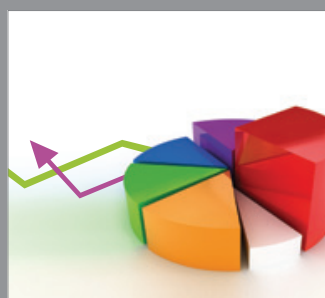

ournal of

Probability and Statistics

Promensencen
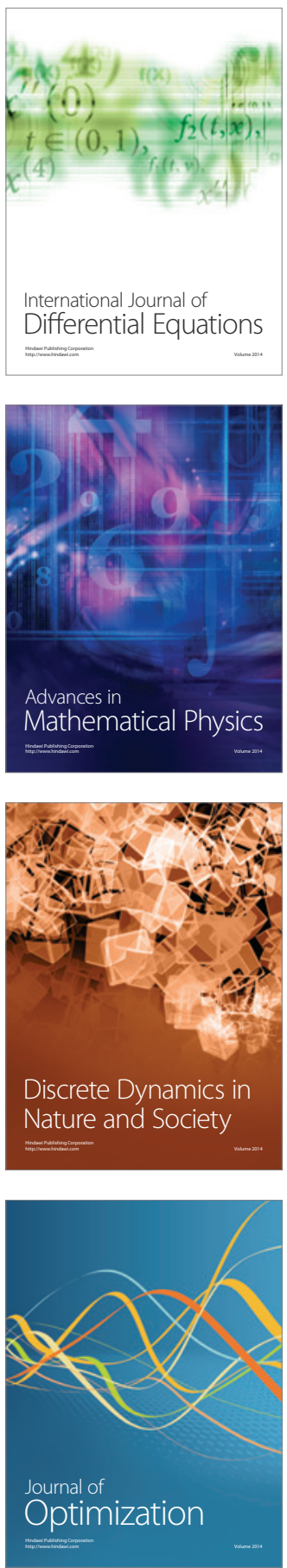